\title{
Four-dimensional imaging and free-energy analysis of sudden pore-filling events in wicking of yarns
}

\author{
Robert Fischer $\odot,{ }^{1,2,3}$ Christian M. Schlepütz $\odot,{ }^{4}$ Dirk Hegemann $\odot,{ }^{5}$ René M. Rossi $\odot{ }^{2}$ \\ Dominique Derome $\left(\mathbb{0},{ }^{6}\right.$ and Jan Carmeliet $\mathbb{0}^{3}$ \\ ${ }^{1}$ Laboratory of Multiscale Studies in Building Physics, Empa, Swiss Federal Laboratories for Materials Science and Technology, \\ Überlandstrasse 129, 8600 Dübendorf, Switzerland \\ ${ }^{2}$ Laboratory for Biomimetic Membranes and Textiles, Empa, Swiss Federal Laboratories for Materials Science and Technology, \\ Lerchenfeldstrasse 5, 9014 St. Gallen, Switzerland \\ ${ }^{3}$ Chair of Building Physics, Swiss Federal Institute of Technology Zurich (ETHZ), Stefano-Franscini-Platz, 5, 8093 Zurich, Switzerland \\ ${ }^{4}$ Swiss Light Source, Paul Scherrer Institut, 5232 Villigen PSI, Switzerland \\ ${ }^{5}$ Advanced Fibers, Empa, Swiss Federal Laboratories for Materials Science and Technology, 9014 St. Gallen, Switzerland \\ ${ }^{6}$ Department of Civil and Building Engineering, Université de Sherbrooke, Sherbrooke, Québec, Canada J1K 2R1
}

(Received 31 December 2020; accepted 13 April 2021; published 10 May 2021)

\begin{abstract}
What are the mechanisms at play in the spontaneous imbibition dynamics in polyethylene terephthalate filament yarns at pore scale? Processes at pore scale such as waiting times between the filling of two neighboring pores, as observed in special irregular porous media, like yarns, may overrule the predicted behavior by well-known laws such as Washburn's law. While the imbibition physics are well known, classic models like Washburn's law cannot explain the dynamics observed for yarns. The stepwise dynamics is discussed in terms of the interplay of thermodynamic free energy and viscous dissipation. Time-resolved synchrotron x-ray microtomography documents water filling at pore scale. Spontaneous imbibition in yarns is characterized by a series of fast pore-filling events separated by long periods of low flux. Four-dimensional imaging allows the extraction of interface areas at the boundaries between water, air, and polymer and the calculation of free-energy evolution. It is found that the waiting periods correspond to quasistable water configurations of almost vanishing free-energy gradient. The distributions of pore filling event sizes and waiting times spread over several orders of magnitude, resulting in the pronounced stepwise uptake dynamics.
\end{abstract}

DOI: 10.1103/PhysRevE.103.053101

\section{INTRODUCTION}

Spontaneous imbibition of a liquid from an unlimited reservoir at atmospheric pressure into a fibrous structure by capillary action is of relevance for medical textiles, moisture management in functional clothing, or production of fiber reinforced materials. Fluid flow in textiles can be anomalous and challenging to describe due to the high complexity of flow paths and interface interactions. Time-resolved x-ray tomographic imaging and thermodynamic energy analysis can shed light on the processes involved. Textiles are three-dimensional (3D) objects produced from quasi-1D linear yarns, for example, by weaving or knitting. Yarns are twisted fiber bundles within which elongated pores form. The fibers can be porous themselves, but we restrict this study to dense fibers with a circular cross section. Textiles inherently state a multiscale problem as pores form also between clothing layers, fabrics, and yarns. The porous medium of interest here is the void space between the fibers inside a yarn and this study focuses on spontaneous imbibition in yarns. Imbibition, as opposed to drainage, is the invasion of a wetting fluid into porous media by replacing a nonwetting fluid. The special case of this process happening by itself without external forcing, e.g., pumping, is called spontaneous imbibition. Classically, this process is also called wicking for textiles and described by Washburn's law [1], which predicts the movement of a saturated water front in a cylinder following a square root of time behavior when gravity can be neglected. The principle physics governing the process is described, for example, in [2], stating that the wetting force is balanced by viscous dissipation. Inertia plays a role for spontaneous imbibition only in the case of particularly-low-viscosity liquids [3]. As long as the wetting force can be approximated in general porous media by its mean and the dissipation factor remains constant, i.e., the flow resistance grows linearly with the traveled distance, spontaneous imbibition follows a square root of time behavior [4]. If these assumptions do not hold due to a more complex pore structure, the approximation is sometimes refined to a higher degree, e.g., by a dual-porosity continuum Darcy-type model [5], or pore-structure-informed methods such as the pore morphology method [6] or pore network models (PNMs) [7-13]. Where Washburn, continuum, and pore network models assume a smooth uptake process and approximate the pore-filling processes in a strong simplification, stepwise water uptake via a series of bursts has been recently reported [14], especially in the context of spontaneous imbibition in yarns [15] and textiles [16]. This stepwise uptake process has been disregarded and its apparent effect on the overall wicking dynamics neglected [1,17-22]. Meniscus arrest and stepwise filling at pore scale can in some cases be attributed to pressure distributions pulling water to neighboring pores [23]. The dynamic PNM [7,9-13] solves 
implicitly the interpore pressure distribution and considers this type of meniscus arrest. However, it cannot explain, for instance, the almost complete halt of the uptake. Remobilization of a pinned meniscus at a geometric singularity or sharp expansion of the pore radius has been attributed to molecular precursor films [14] and diffuse interfaces [24]. Our in-depth microscopic analysis presented in this paper leads to a more precise understanding of only seemingly pinned menisci that slowly trespass zones of capillary quasiequilibria. This explanation also applies for experimentally observed transition times in real and singularity-free porous media [14]. Due to the specific pore structure of yarns, these pores can undergo rapid filling and transport large amounts of liquid over long distances, highlighting the importance of understanding the pore-scale processes [15]. This process is different from Haines jumps where external pressure breaches local equilibria leading to rapid liquid reconfigurations until the subsequent equilibrium is reached [25].

Numerical approaches to predict two-phase flow in pores include volume of fluid computational fluid dynamics methods [26], lattice Boltzmann methods [12,13,27,28], and even molecular-dynamics simulations [29]. While these methods give valuable fundamental information, the computational requirements to simulate systems as complex as yarns are still unattainably high. Experimentally, microfluidic models are employed to study the pore-filling process in well-defined porous structures and to allow the use of optical methods due to the usually transparent materials, but are often limited to 2D images [25]. Experimental measurements of the liquid configuration evolution are in general difficult to obtain with conventional imaging methods, e.g., high-speed photography, in porous media due to their complex three-dimensional and opaque structure. Singh et al. [30] employed time-resolved synchrotron x-ray tomographic microscopy (XTM) to study Haines jumps in packed spherical beads. Another good example for investigating liquid interfaces with microtomography is the work by Lin et al. [31], where they determined the fluid interface curvature at the static equilibrium to ultimately obtain the relation between saturation and capillary pressure in porous media.

Different from these experiments of flow in porous media with external forcing, Piovesan et al. [32] studied capillary rise in microfluidic channels and Parada et al. [15] investigated spontaneous water imbibition into hydrophilic textile yarns highlighting the stepwise filling process. The principal use of imaging, especially time resolved, is to get qualitative insights into otherwise hidden processes $[15,33]$. Several studies on imaging two-phase flow in porous media revealed the difficulty of using the apparent contact angle to determine the wetting force acting on the fluid because of the wide variability of measured contact angles $[31,34,35]$. When the equilibrium contact angle is known, the image data provide additional information so that the measurement of interface areas allows the calculation of the wetting force via the free-energy gradient. To obtain accurate quantitative data, the grayscale images first have to be converted to phase segmented data. Developments of machine-learning-based [36] and other clustering-based [32] segmentation methods allow improved spatial resolution on static 3D images over simple thresholding. To better harvest the temporal information of $4 \mathrm{D}$ image data, Parada et al. [15] proposed a segmentation method based on the temporal evolution of the image data instead of segmenting a series of static images. Combining temporal and spatial methods is necessary to make ideal use of dynamic XTM. A fluid interface can indeed be obtained from XTM as demonstrated, e.g., by Lin et al. [31] using a triangulated mesh extracted from the voxelized XTM data.

In this paper, XTM is used to image the spontaneous imbibition of water in polyethylene terephthalate yarns showing stepwise filling events at pore scale. This work intends to study the evolution of water-air interfaces and water-wet-solid areas to understand the stepwise flow during spontaneous imbibition. The evolution of free energy versus time is determined actually showing stepwise decreases of free energy during sudden filling events. These sudden variations in free energy are caused by the specific pore structure of yarns and the evolution of the water configuration during imbibition.

First, we describe in general the change in free (surface) energy during spontaneous imbibition in yarn pores. Then the materials and imaging, processing, and postprocessing methods are presented together with the analysis approach. Exemplary qualitative image results are given before discussing the quantitative findings by free-energy analysis which are compared to similar processes in other fields. Finally, conclusions are drawn with an outlook for future research.

\section{FREE (SURFACE) ENERGY DURING SPONTANEOUS IMBIBITION IN YARN PORES}

Spontaneous imbibition of water in yarn pores is a twophase flow phenomenon where water is the wetting fluid and air the nonwetting fluid. The solid area wet by water is denoted by the wet surface $A_{w s}$ and the water-air interface by $A_{w a}$. Because the capillary and Bond numbers for yarn pores are in the range of $10^{-6}$, we may conclude that we are in the capillary regime and gravity does not play a role. Since the imbibition process is accompanied by low water velocities (low Reynolds number, i.e., in the range of $10^{-2}$ ), the flow will be laminar and inertia can be neglected [3]. Therefore, spontaneous imbibition is controlled only by capillary forces and viscous dissipation. The energy flux balance equation neglecting contributions of kinetic energy and inertia effects then reads [26,37]

$$
-\Phi=\frac{d F}{d t},
$$

where $F$ (in $\mathrm{J}$ ) is the free energy and $\Phi$ (in $\mathrm{J} / \mathrm{s}$ ) is the viscous dissipation rate, which equals [38]

$$
\Phi=R Q^{2},
$$

with $R$ (in $\mathrm{Pa} \mathrm{s} / \mathrm{m}^{3}$ ) the flow resistance and $Q\left(\right.$ in $^{3} / \mathrm{s}$ ) the volume flux equal to $d V / d t$. Note that the flow resistance $R$ for a pore situated far from the free water surface is not only the resistance of the pore itself $R_{p}$, but also includes the resistance from the free water surface to the pore entry, $R_{e}$. This means that the flow resistance is $R=R_{e}+R_{p}$. Equations (1) and (2) show that a reduction of free energy is accompanied by viscous dissipation, whose magnitude is governed by the magnitude of flux $Q$ and pore resistance $R$. Equation (1) can be 


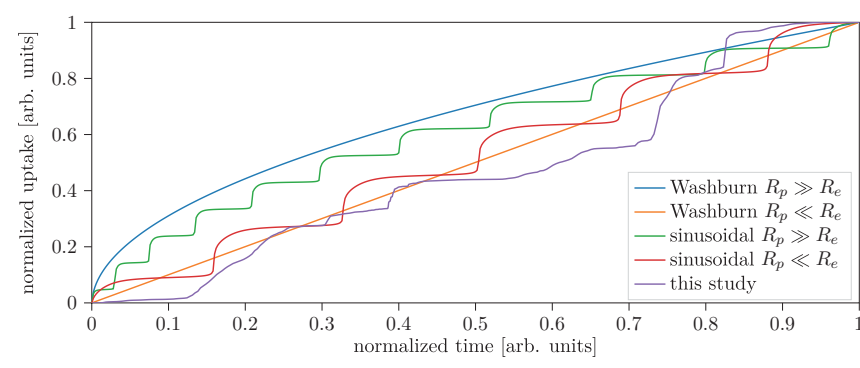

FIG. 1. Comparison of different normalized uptake curves depending on water-air interface area change and external upstream resistance: Washburn [1] $R_{p} \gg R_{e}, d A_{w a}=0$ and $d A_{w s}=$ const $>$ 0; Washburn [1] $R_{p} \ll R_{e}, d A_{w a}=0$ and $d A_{w s}=$ const $>0$; sinusoidal [39] $R_{p} \gg R_{e}, d A_{w a} \neq d A_{w a} \neq$ const; sinusoidal [39] $R_{p} \ll$ $R_{e}, d A_{w a} \neq d A_{w a} \neq$ const; this study, experimental example for a yarn in this study.

further specified assuming that the free energy only depends on the volume $V$ of pore space being filled by water

$$
-\frac{\partial F}{\partial V} \frac{d V}{d t}=R Q^{2}
$$

or

$$
-\frac{\partial F}{\partial V}=R Q
$$

where the left-hand side equals the capillary pressure $p_{c}$. Rewriting Eq. (4), we get

$$
Q=\frac{p_{c}}{R}
$$

which equals Darcy's law at the pore level. For nonreactive and isothermal flow, the infinitesimal increment of free energy $F$ can be written in terms of surface energy as [26]

$$
\begin{aligned}
d F & =\gamma_{w a} d A_{w a}+\gamma_{w s} d A_{w s}+\gamma_{s a} d A_{s a}, \\
\gamma_{w a} \cos \vartheta & =\gamma_{s a}-\gamma_{w s}, \\
d A_{w s} & =-d A_{s a}, \\
d F & =\gamma_{w a}\left(d A_{w a}-d A_{w s} \cos \vartheta\right),
\end{aligned}
$$

where $\gamma_{w a}$ is the water-air surface tension, $d A_{w a}$ and $d A_{w s}$ are the infinitesimal increments of the water-air interface and wet (water-solid) surface area, respectively, and $\vartheta$ is the equilibrium contact angle. In the derivation of the free-energy increment (6), Young's relation is used. While Washburn's square root law over time is a special case [see the Appendix and Eq. (A3)], the presented formulation is more universal. The equations above allow us to interpret flow in pore systems by means of changes in free energy $d F$, related to flow resistance $R$ and the resulting flow $Q$. When $d F=0$, no flow occurs. Flow can only occur when $d F<0$. Assuming spontaneous imbibition in a capillary with constant cross section (Fig. 1, Washburn) and that the meniscus does not change shape, $d A_{w a}$ is equal to zero. The decrease in free energy is then governed only by the change in water-solid area $d A_{w s}$, which is always positive during spontaneous imbibition. In the case that $d A_{w s}$ is constant, the decrease in free energy will be constant. When the pore resistance is also constant, the flux of water will be constant and we obtain a linear uptake over time. A constant flow resistance can be assumed when the pore entry resistance $R_{e}$, which is always constant, is very large compared to the resistance of the pore itself, which changes with uptake height (Fig. 1, Washburn $R_{p} \ll R_{e}$ ). A high pore entry resistance occurs when the pore is located far from the free water surface, e.g., in the middle of a porous medium and the entire path to reach this pore has to be considered. When the pore entry resistance is however negligible, the pore resistance $R$ is not constant and increases linearly with filling height, resulting in a classical square root uptake behavior (Fig. 1, Washburn $R_{p} \gg R_{e}$ ). For single pores $\left(R_{e}=0\right)$ with large cross section (coarse pores), the increase in $A_{w s}$ is large, while the flow resistance $R=R_{p}$ is low, resulting in large fluxes $Q$. In contrast, fine pores showing a high pore resistance and lower decrease of free energy show lower fluxes. In pore systems with varying cross section, the change in wet surface $d A_{w s}$ is positive but its magnitude can vary during the uptake process, while $d A_{w a}$ can be both positive and negative. Figure 1 sinusoidal gives the example of a tube whose radius oscillates with $r=r_{0}+0.5 r_{0} \sin \left(2 \pi h \lambda^{-1}\right)$ [39]. When $d A_{w a}$ is negative, which is the case for narrowing pores, the decrease in free energy is large, but because the resistance increases for narrowing pores, the flux will be lowered. In the case of widening pores, $d A_{w a}$ is positive and increases, leading to a lower decrease in free energy, and as such yielding a slower uptake, even when the resistance of the pore is low. In the case of large and constant $R_{e}$ (Fig. 1 , sinusoidal $R_{p} \ll R_{e}$ ), the flux variation depends entirely on the water-air interface evolution. Note that, due to the opposite signs in parentheses in Eq. (6), the possibility of close-to-zero free-energy gradient exists. A temporary local energy quasiminimum is reached for almost zero gradient and the water obtains a quasistable configuration for some time and capillary uptake almost stops (Fig. 1, sinusoidal and this study). However, since the free-energy gradient is not totally zero, a small flux can still occur for long periods due to very slow variations of $A_{w s}$ and $A_{w a}$, referred to here as the waiting period. After this waiting period, a sharp decrease in free energy may result in a very fast pore-filling event showing a large volume flux. In conclusion, in regular pore systems where the pore sections remain almost constant, the decrease in free energy will be rather constant, and when those pores show a rather constant resistance, the flux of water will be almost constant. In irregular pore system with highly varying pore cross sections, high water volume can be transported in zones of high free-energy gradient during sudden pore-filling events, while it may take long times to traverse the zones of almost vanishing free-energy gradient. The consequence is a stepwise wicking dynamic consisting of series of filling events separated by long waiting times (see Fig. 1, this study, purple curve). In Sec. IV we will study the stepwise pore filling in yarn pores by analyzing the time evolution of free (surface) energy.

\section{EXPERIMENTAL METHOD}

\section{A. Materials}

Yarns are produced by twisting cylindrical filaments, made of polyethylene terephtalate (PET) (typically called polyester in the context of textiles) with a diameter of $55 \mu \mathrm{m}$ (obtained from Monosuisse AG, Emmenbrücke, Switzerland). 


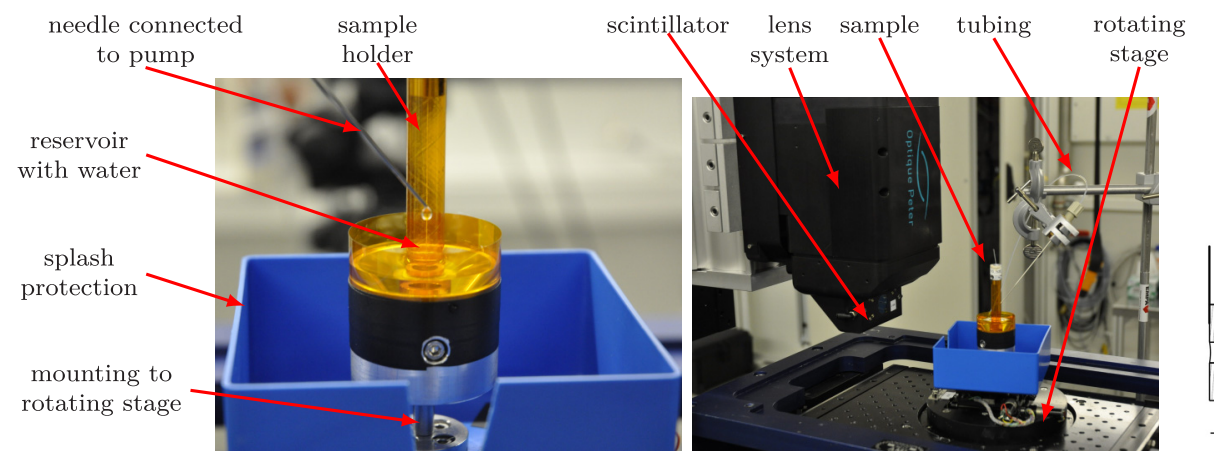

(a) (b)

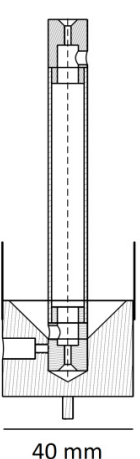

(c)

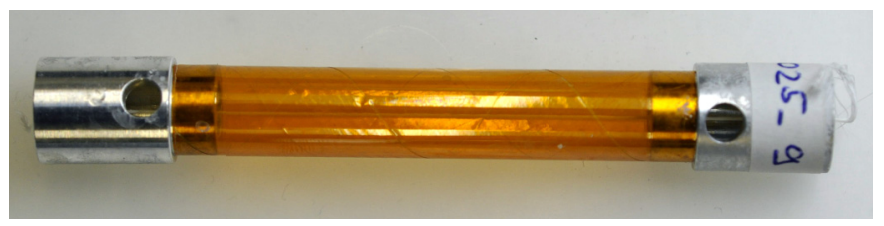

(d)

FIG. 2. Experimental setup showing (a) the sample holder mounted to the water-filled reservoir and (b) the whole ensemble on the rotating stage in front of the detector system. (c) Drawing of the holder with the yarn position as the dashed line. (d) Close-up of the sample holder.

Plasma-enhanced chemical vapor deposition (PECVD) is applied to ensure constant and permanent wettability of the filaments [40,41]. A detailed description of the applied PECVD process can be found in the Appendix. It is difficult to accurately measure the wettability on thin filaments, because Young's contact angle describing wettability is defined for a flat and ideal solid surface only. The filament surface curvature is not negligible when obtaining the apparent geometric contact angle with optical means. Force-based wettability measurements like the Wilhelmy method require unreasonable sensitivity to detect the minimal force produced by wetting the small filament perimeter. Experiments on flat PET plates under equivalent PECVD conditions suggest a resulting Young's contact angle with water in air of $48^{\circ} \pm 5^{\circ}$. Bundles of 32 filaments and $0.5 \mathrm{~m}$ length are threaded through sample holders and placed in a twist tester (Zweigle D314, Reutlingen, Germany), while the holders cannot come in contact with the bundles. While applying a tension of $2.5,10$, or $30 \mathrm{mN} /$ tex ( 1 tex $=1 \mathrm{~g} / 1000 \mathrm{~m}$ ), the filament bundles are slowly spun to yarns of 200 twists per meter. To preserve the obtained mechanical state, the pinholes of the sample holder caps are filled with a gel glue containing cyanoacrylate (superglue, UHU $\mathrm{GmbH}$, Bühl, Germany) fixing in place the yarn in the center of the holder. Once the glue has cured, the yarn is cut at both end of the holders. Figure 2 shows the experimental setup, with the sample holder mounted in the water-filled reservoir in Fig. 2(a), the whole ensemble on the rotating stage in front of the detector system in Fig. 2(b), and the section drawing of the setup in Fig. 2(c). The experimental setup is custom designed for this experiment. It consists of an aluminum reservoir with an extended wall made of Kapton foil and a sample holder made with a 10-mm-wide Kapton tube with two aluminum end caps. Kapton provides high transparency to x-ray and high mechanical stability, allowing us to maintain the yarn in the set tension and twist. The cylindrical caps have a $1-\mathrm{mm}$ pinhole along their rotation axis through which the yarn is inserted and glued in place as mentioned above. Additionally, large holes on the side, one at the bottom and one at the top, allow the entry of water and the exit of air.

\section{B. Synchrotron x-ray tomographic microscopy}

Imaging is performed at the tomographic microscopy and coherent radiology experiments (TOMCAT) beamline of the Swiss Light Source (SLS), Paul Scherrer Institut, Villigen PSI, Switzerland [42], where recent development in fast tomography permits us to acquire XTM data with sufficient temporal and spatial resolution to study the time evolution of water interfaces in yarn pores. The x-ray beam is provided by a 2.9-T superbend at a critical energy of $11.1 \mathrm{keV}$ installed in the SLS ring. A white beam filter consisting of $20 \mathrm{~mm}$ of pyrolitic carbon plus $75 \mu \mathrm{m}$ of molybdenum is used to filter out $x$ rays with low energies as well as attenuating those above $20 \mathrm{keV}$ in energy. The resulting spectrum has a pronounced energy peak between 15 and $20 \mathrm{keV}$, which produces a good imaging contrast for the water-filled structure while avoiding any noticeable beam-induced bubble formation in the liquid. The $\mathrm{x}$ rays penetrate the sample and hit at a distance of $150 \mathrm{~mm}$ a $150-\mu \mathrm{m}$-thick LuAl:Ce scintillator where they are converted into visible light. The relatively large propagation distance between the sample and scintillator provides for a good degree of edge enhancement due to the partially coherent X-ray beam, allowing the application of propagation-based phase contrast algorithms to boost the image contrast. The scintillator image is magnified by a custom-made macroscope [43] and recorded by a complementary metal-oxide semiconductor chip inside the GigaFRoST [44] camera. This setup allows the acquisition of one good quality image per millisecond and effective pixel size of $2.75 \mu \mathrm{m}$ in a full frame of $2016 \times 2016$ pixels. Due to the linear shape of the yarns, 
TABLE I. Applied parameters during the different processing steps. Numeric values are chosen as best educated guesses under subjective inspection of the image data.

\begin{tabular}{|c|c|c|c|}
\hline Step & Method & Dimension & Parameter \\
\hline 1 & $\begin{array}{l}\text { Paganin-filtered } \\
\text { reconstruction }\end{array}$ & $\begin{array}{l}3 \mathrm{D} \text { per } \\
\text { time step }\end{array}$ & $\begin{array}{c}E=19.5 \mathrm{keV}, \delta=7.5 \times 10^{7}, \beta=1 \times 10^{-9} \\
D_{p}=0.15 \mathrm{~m}, \min =-5 \times 10^{-7}, \max =4 \times 10^{-6}\end{array}$ \\
\hline 2 & intensity correction & $2 \mathrm{D}(x y)+$ time & first slice $(t=0)$ as reference \\
\hline 3 & registration & $2 \mathrm{D}(x y)+$ time & rigid body, first slice as reference \\
\hline 4 & Weka segmentation & $2 \mathrm{D}(x y)$ & filters: Gaussian blur, Sobel, difference of Gaussians, Hessian, membrane projections \\
\hline 5 & water segmentation & time & $\begin{array}{c}\text { water threshold, 6000; transition threshold, } 1500 ; \text { receding threshold, }-2000 ; \\
\text { low-pass filter: frequency, } 0.1 ; \Delta t=1.2\end{array}$ \\
\hline 6 & pore segmentation & $3 \mathrm{D}$ & disconnection, $0.7 ; x$ size, $1.5 ; y$ size, $1.5 ; z$ size, 1 \\
\hline 7 & interface extraction & 3D per time step & Taubin smooth: $k=0.1, \lambda=0.6307$, ten iterations \\
\hline
\end{tabular}

the images are promptly cropped to $240 \times 2016$ pixels to reduce data size. For a sample rotating at $1 \mathrm{~Hz}, 500$ projections per $180^{\circ}$ are recorded, sufficient for fully acquiring one high quality tomographic scan in half a second. Prior to the experiment, 30 dark field (no beam) and 50 flat field (with beam, no sample) images are recorded to correct for image heterogeneity.

\section{Running of the experiment}

The sample is fixed in the reservoir and placed on the rotating stage at TOMCAT as displayed in Fig. 2. To start the acquisition, the sample is accelerated to $1 \mathrm{~Hz}$ and then $14.2 \mathrm{ml}$ of water are released via a remotely controlled micropump and the camera starts recording simultaneously. Due to limitations in the amount of data size that can be acquired per sample and the expected duration of the wicking process of around $30 \mathrm{~min}$, the temporal resolution is spread strategically. Different sequences were acquired, but the acquisition scheme was usually one scan every $6 \mathrm{~s}$ for $10 \mathrm{~min}$, then $1 \mathrm{scan} / \mathrm{s}$ for $2 \mathrm{~min}$, and again one scan every $7 \mathrm{~s}$ for another $18 \mathrm{~min}$, for a total of 30 min of water uptake and 361 tomographic data sets. The intention of this strategy is to capture the whole uptake process within a reasonable amount of data while also incorporating a short time interval with the maximal time resolution. The field of view (FOV) is framed to include the free water surface in the reservoir and around $4 \mathrm{~mm}$ of the yarn. Twelve samples per tension value are tested. After the samples have dried, the experiments are repeated for those that showed water uptake. Finally, only those acquisitions showing clear water uptake are analyzed (15: four with $2.5 \mathrm{mN} /$ tex, seven with 10 $\mathrm{mN} /$ tex, and four with $30 \mathrm{mN} /$ tex).

\section{Image processing}

\section{Reconstruction and image filtering}

The acquired edge-enhanced absorption images are reconstructed to generate 3D grayscale pixel data [Figs. 3(a) and 3(c)]. To enhance the minimal material absorption contrast between water and polymer, a phase filter is employed to the projections [45], which is then followed by a standard tomographic image reconstruction with the gridrec algorithm [46]. The reconstruction parameters are optimized for one sample and then applied to the rest of the data set
(Table I). The following processing steps are performed using in-house-developed PYTHON scripts employing standard libraries [47-50] and on 2D slices if not stated otherwise.

The flat water surface in the reservoir surrounding the fiber bundle refracts the grazing incident x-ray light very strongly, leading to a huge edge-enhancement effect of the air-water interface, which mostly obscures the x-ray transmission image of the yarn itself close to the height of this surface. To correct for this artifact, the gray values for each scan in the time series are normalized in reference to the dry sample by subtracting the slicewise difference of the average image intensity.

Although the beamline is equipped with a high-precision rotating stage, the sample differs in its position inside the images of the time series in the range of a few pixels mostly by rotation. Rigid-body registration is required and performed using the TurboReg algorithm [51] in the PYTHON/C++ port by Lichtner [52].

\section{Air-water-polymer segmentation}

The progress in image acquisition hardware, especially the macroscope [43], allows the segmentation of the polymer phase in the dry state effectively providing a mask for the interfilament void space to enhance the segmentation accuracy. As the polymer phase remains immobile during water uptake, the first scan of the still dry sample can be used as reference for the yarn geometry. The 3D grayscale data are segmented into a binary image of air and polymer by the application of the Weka machine learning plug-in [36] for IMAGEJ (Fiji) [53]. In two randomly selected slices (orthogonal to the yarn axis), the circular filament cross sections are manually marked and passed to the algorithm to train a classifier using the image filters listed in Table I. This classifier is then applied to all 2016 slices to obtain a probability map of the image [Fig. 3(b)], where $100 \%(0 \%)$ corresponds to pixels certainly belonging to the polymer (air) phase. Pixels with a probability value above $55 \%$ are then assigned to the polymer phase [Fig. 3(c)], outperforming any threshold segmentation method.

As already described by Parada et al. [15], standard segmentation methods for the water phase do not give satisfactory time-resolved results due to the high noise ratio with little polymer-water contrast and we refine their method as described in the following. The time series of the gray value per voxel inside the void space is searched for its 


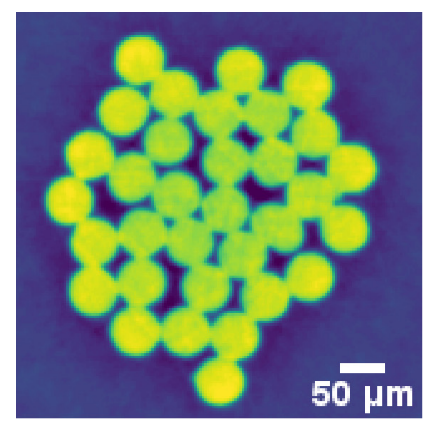

(a)

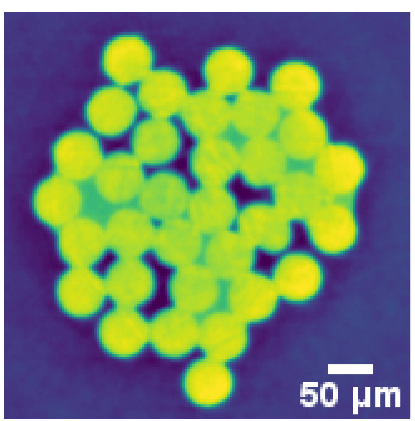

(d)

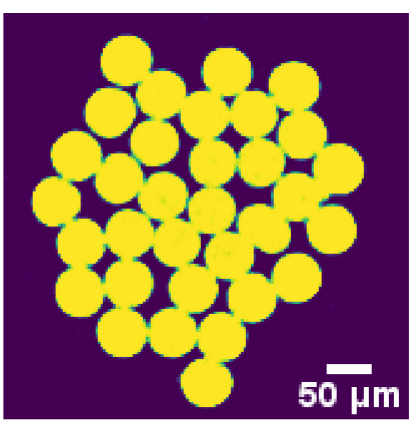

(b)

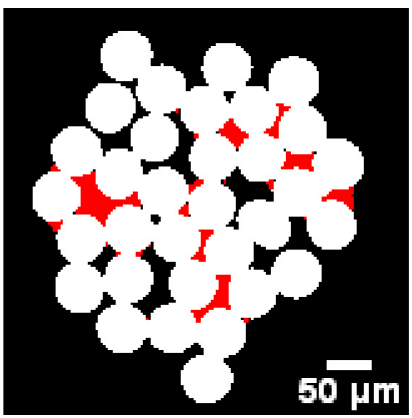

(e)

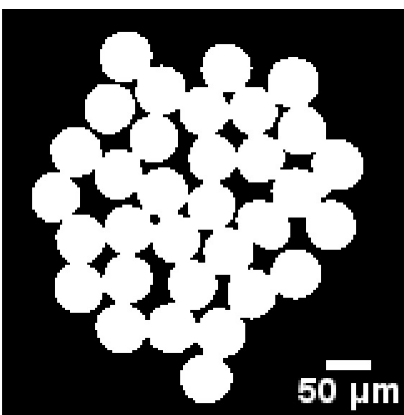

(c)

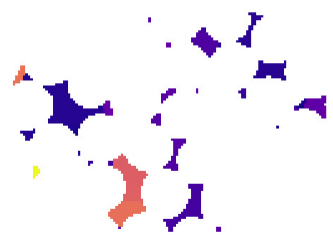

$50 \overline{\mu m}$

(f)

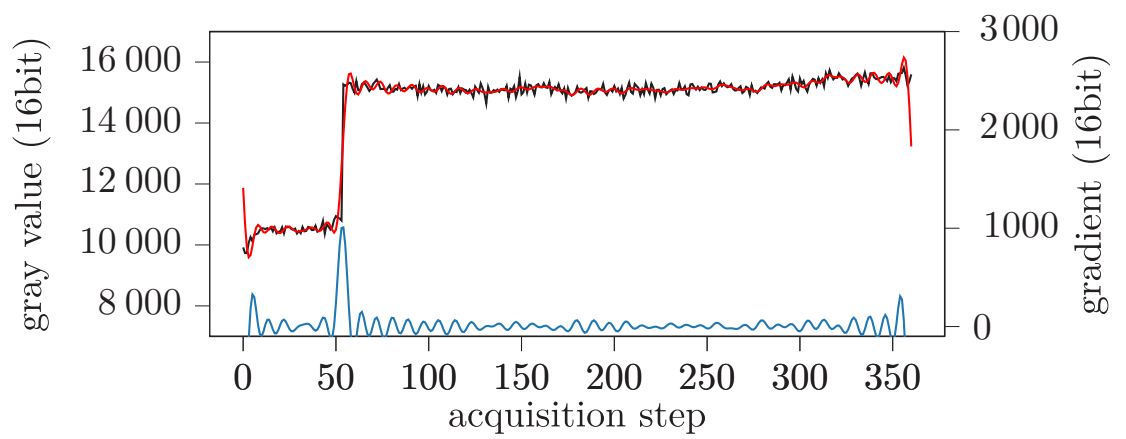

(g)

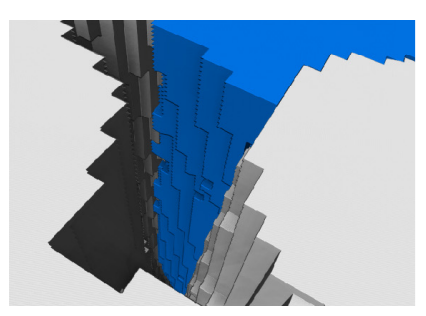

(h)

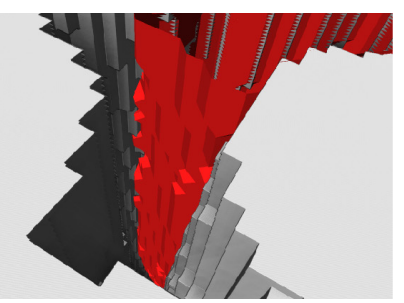

(i)

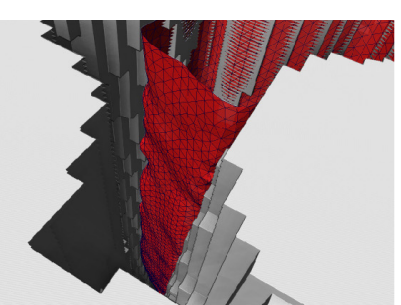

(j)

FIG. 3. Illustration of image processing: (a) raw reconstructed image without water, (b) corresponding machine learning enhanced phase contrast image, (c) fiber-air segmented image, (d) raw reconstructed image with water, (e) water-fiber-air segmented image, (f) time encoded segmented image for voxel air to water transition, (g) water segmentation in a voxel by maximal gray value gradient (black, gray value over time; red, low-pass filtered; and blue, gradient); and (h)-(j) 3D view of the procedure to obtain triangulated water interface mesh, (h) voxel data, (i) Leviner mesh, and (j) smooth mesh (white, fiber; blue, water; and red, water interface).

water-filling event characterized by clear transitions in gray value from dark (air) to bright (water). First, a low-pass filter is applied to remove high-frequency noise and the gradient of this curve is determined [Fig. $3(\mathrm{~g})]$. Then the time at which the gradient in gray value is maximum is determined and if there is a clear difference between the mean gray values in intervals before and after, this time is taken as the time when water has reached this voxel. The rare case of water receding from a voxel is considered by testing the inverse transition at the time step of most negative gradient 
happening after the wetting transition. The case of rewetting is not observed.

The final segmented data consists of three 3D data arrays, one Boolean for the segmented polymer phase [Fig. 3(c)] and two 16-bit integer sets where the voxel value is the time step of wetting or receding transition, respectively [Fig. 3(f)]. The acquisition time can then be related to physical time. Due to the availability of an accurate polymer segmentation and enhanced image quality, the sensitivity for water detection can be increased compared to our previous study [15] with the same segmentation method barring the polymer mask. The spatial uncertainty of the polymer phase is estimated to around one to two voxels, while the effective temporal resolution for the transition of a voxel from air to water is also one to two time steps.

\section{Data postprocessing and analysis of water uptake}

The temporal evolution of the total water content of the yarn in the FOV can be readily extracted from the segmented data since the voxel value also denotes the time step when water is reaching the specific voxel. One has to count the water voxels per time and/or height and multiply the value with the voxel volume $(2.75 \mu \mathrm{m})^{3}$. The volume flux $Q$ is the temporal gradient, i.e., the second-order central difference, of the volume. The next step is to consider the water movement pore by pore. This requires a pore segmentation of the yarn. The division into separate pore bodies depends on the employed experimental method and is often problematic [54]. Standard pore segmentation processes like the maximum ball or watershed algorithm result in a strong oversegmentation when applied to yarns. A restricted watershed algorithm [55], implemented in JAVA and provided as an IMAGEJ plug-in, segments the 3D void space outlined as a convex hull around the fibers in long and narrow pores separated at concave gaps. Contrary to a conventional watershed, this algorithm limits the segmentation by introducing a constraint during the erosion of the pore space and the erosion distance $d$ needs to be smaller than the maximum distance from the solid surface $r$ multiplied by a disconnection factor $k$ [55]. Here $k=1$ recovers the conventional watershed and $k=0$ results in no pore segmentation. Similar to [55], a disconnection factor $k$ of 0.7 was found to provide a meaningful pore segmentation as a best possible fit by visual judgment (Fig. 4).

The wet surface $A_{w s}$ is determined by first applying a binary dilation to the image data of the fiber phase and then extracting the overlap with the water phase giving a mostly one-voxel-wide virtual solid-water interface phase. The marching cube algorithm of Lewiner et al. [56] provides a triangulated surface mesh which is then smoothed by Taubin's volume conserving algorithm [57]. The resulting mesh is effectively a double-faced thin sheet between the solid and the water phases. The wet surface is therefore the mesh area of one face.

To tackle the noisy interface between air and water, a slightly different method is employed to determine the waterair interface. The algorithms of Lewiner et al. and Taubin are directly employed to the surface of the water phase [Figs. 3(h)-3(j)]. The fiber phase is dilated and all mesh vertices inside this padded volume are labeled as a wet solid surface, i.e., water surface touching the solid. Because the pore segmentation also separates the water into pore water bodies, the pore boundary can constitute a virtual interface to water in a neighboring pore. Vertices in contact with water bodies of neighbor pores are labeled in the same way as they would be at the solid. The water-air interface area is therefore the total water surface area less the virtual interface and the surface touching the solid. Knowing the water-solid area $A_{w s}$ and water-air interface area $A_{w a}$, the free-energy increment $d F$ per time step $d t$ can be determined according to Eq. (6). For this calculation, we used a water-air surface energy of $72 \mathrm{mN} / \mathrm{m}$ and a contact angle of $48^{\circ}$. Knowing the volume flux $Q$ and time gradient of the free energy $\frac{d F}{d t}$, the flow resistance $R$ can be determined using Eqs. (1) and (2). We recall that $R$ is not the pore resistance but the total flow resistance, which is the sum of the pore entry resistance $R_{e}$ and pore resistance $R_{p}$ itself.

\section{RESULTS}

\section{A. Description of imaging data}

The algorithm proposed in Sec. III D 3 is used to separate the void space into long and narrow pore bodies as displayed in Fig. 4. Figure 4(a) shows an example of a grayscale tomographic horizontal slice before pore segmentation. Figures 4(b)-4(d) show one cross and two longitudinal sections of labeled pores, while Fig. 4(e) gives a 3D image of the pore space. The labeled images allow the analysis of several geometrical pore properties, notably shape factor, aspect ratio, variance of cross-sectional area, and connectivity, i.e., the number of neighbors. The median shape factor describes the pore angularity and is defined as the median along the yarn axis of the ratio ration of the cross-sectional area to the square of its perimeter, having its maximum for a circle with $G_{\max }=$ $\frac{A}{P^{2}}=\frac{1}{4 \pi} \approx 0.08$. The aspect ratio is defined as the ratio of the major axis of the $3 \mathrm{D}$ pore object to the square root of the median cross-sectional area. The probability density graphs of these six parameters are displayed in Fig. 5 for the three yarn types, each under a different tension. In total, 988 pores are analyzed, 234 for $2.5 \mathrm{mN} /$ tex, 469 for $10 \mathrm{mN} /$ tex, and 285 for $30 \mathrm{mN} / \mathrm{tex}$. The concave shape of the interfilament pores results in angular pores with many corners or grooves at the filament contacts [Fig. 5(a)]. Despite the high aspect ratio up to 110 [Fig. 5(b)] of the long and narrow pores, the change of cross-sectional area is limited (very little relative area variance above $50 \%$ and mostly $25 \%$ ), indicating homogeneous tubelike pore bodies [Fig. 5(c)]. The connectivity of the pore network is for most pores small with a peak at two, but can increase for some pores to ten and more [Fig. 5(d)], which at least in some cases may be due to spurious pixels labeled as separate pores. There is a strong variance for both pore cross sections [119\%, Fig. 5(e)] and lengths [97\%, Fig. 5(f)] over the set of pores. Typical pores have a diameter in the range of $10 \mu \mathrm{m}$, while the height reaches up to several 100 $\mu \mathrm{m}$. A regular pore is a long and narrow capillary showing several concave faces forming several pointy corners (Figs. 4 and 6). The backbone of the capillary is parallel to the yarn axis, while showing a slight pitch following the yarn twist (Figs. 4 and 6), and the variation of the cross section over 


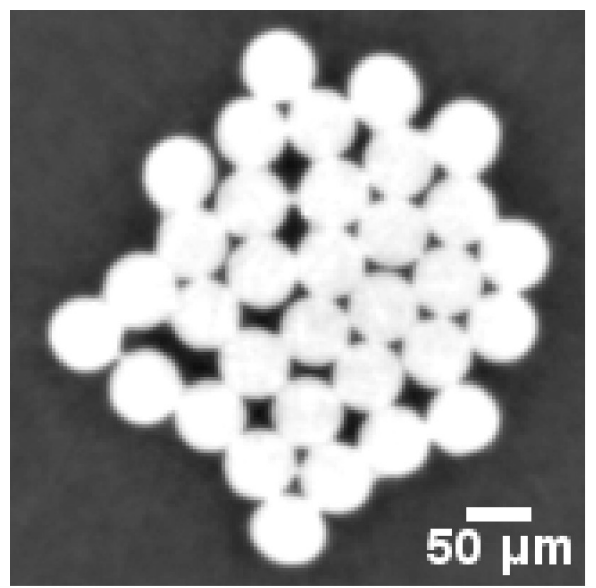

(a)

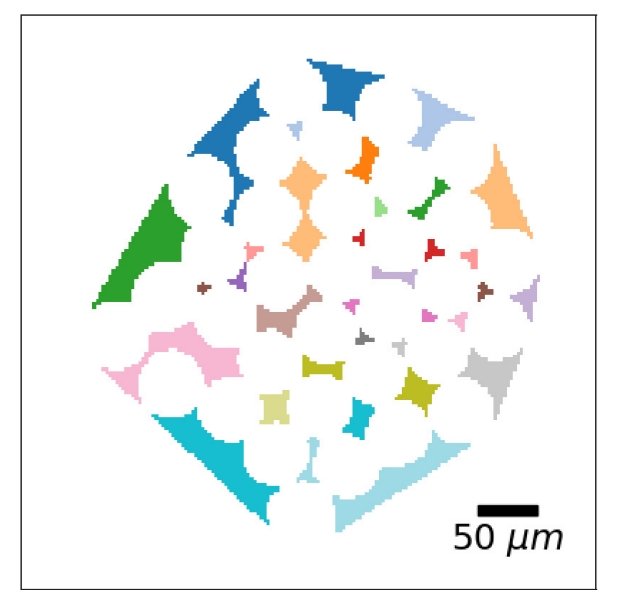

(b)

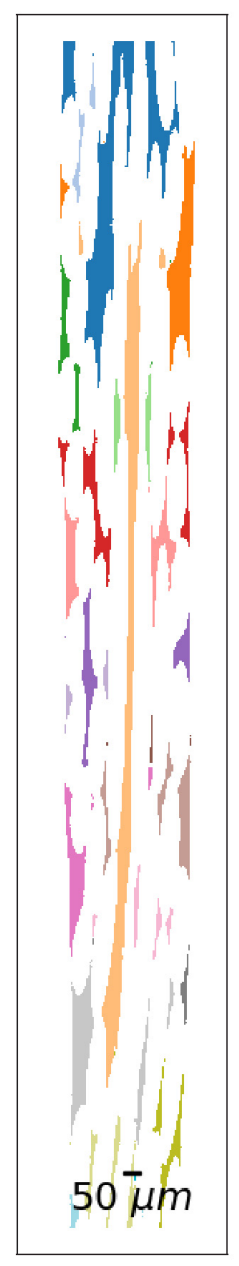

(c)

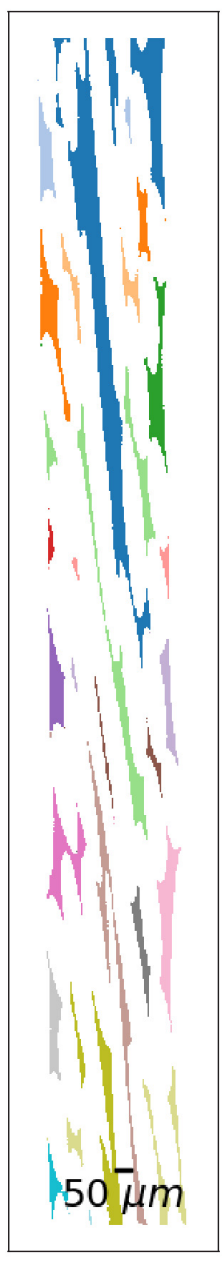

(d)

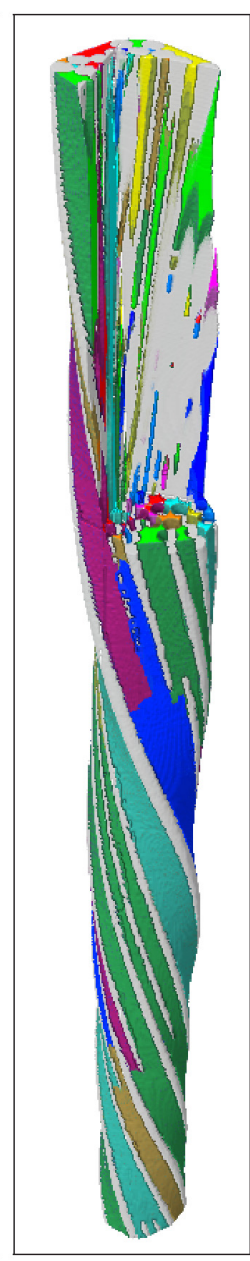

(e)

FIG. 4. Visualization of the yarn pore structure: (a) grayscale tomographic horizontal slice, with the corresponding labeled pore space in the (b) horizontal, (c) longitudinal, and (d) frontal planes. (e) The 3D projection. Note that, for the sake of improved readability, label colors do not necessarily correspond to the same pores.

height is with $22 \%$ low [Fig. 6(a)]. Some pores show unusual shapes. As an example, the pore in Fig. 6(b) starts from the bottom as four separate capillaries that subsequently merge to a wide quadruple-conjoint capillary to diverge again at the top into three capillaries. This pore has a larger cross-section variance $(68 \%)$ with a large pore volume at a maximum cross section of $2700 \mu \mathrm{m}^{2}$ and larger than $1500 \mu \mathrm{m}^{2}$ over $0.75 \mathrm{~mm}$ in the middle, connecting different smaller pores (minimum at $53 \mu \mathrm{m}^{2}$ ). Pore connections can occur in a variety of ways [see, for example, Fig. 6(c)]. Although the number of studied pores is small, the system shows a high variability in pore sizes and shapes.

\section{B. Spontaneous imbibition at yarn scale}

The imbibition process of 15 yarns of 32 cylindrical filaments of $55 \mu \mathrm{m}$ diameter at three different tension values are imaged and analyzed (see Table II). The cumulative water volume uptake of all samples and the volume flux for three samples are displayed in Fig. 7. The first observation is the wide variation in imbibition curves even between yarns sub- jected to the same tension. The final water volume uptake also differs by $70 \%$, with a minimum of $1.9 \times 10^{-12} \mathrm{~mm}^{3}$ and a maximum of $4.8 \times 10^{-11} \mathrm{~mm}^{3}$. Some samples show a steep initial rise before easing into a slow filling process up to a final water volume, while other samples only show minimal water uptake during an initial waiting period in the range of at least $200 \mathrm{~s}$. All curves in Fig. 7(a) contain multiple steps of variable increase in water volume. The flux over time curves in Fig. 7(b) is characterized by a series of peaks of high flux $\left(Q>0.5 \times 10^{-13} \mathrm{~m}^{3} \mathrm{~s}^{-1}\right)$, indicating strong pore-filling events separated by periods of quasistagnation showing low flux, referred to as the waiting period. As a result, the greater part $(64 \pm 21 \%)$ of the water volume is actually transported during the pore-filling events. Attempting to fit Washburn's square root over time law to the mean uptake curve of all samples results in a very small pore radius of $138 \mathrm{pm}$, which is not in agreement with observed real pore sizes in the range of $10 \mu \mathrm{m}$. The imbibition dynamics expected from the classic Washburn model does not take into account the stepwise dynamics and waiting times, which control the uptake process. As will be shown in the following, filling processes with 


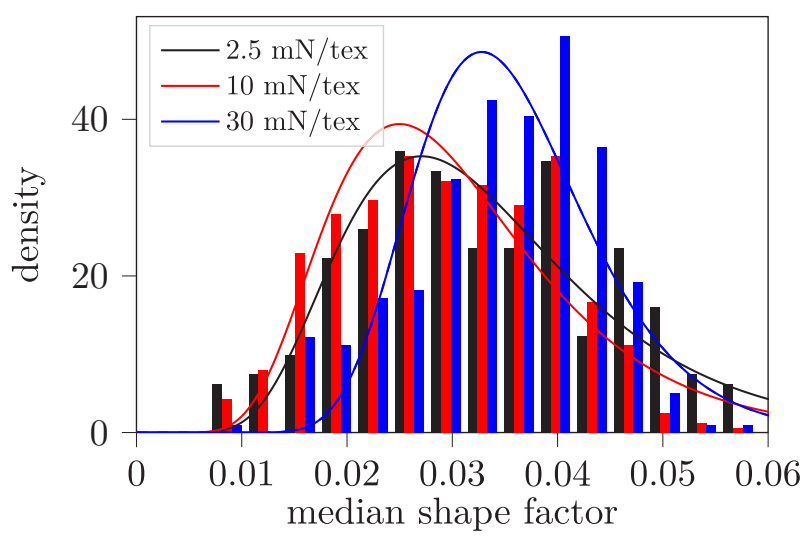

(a)

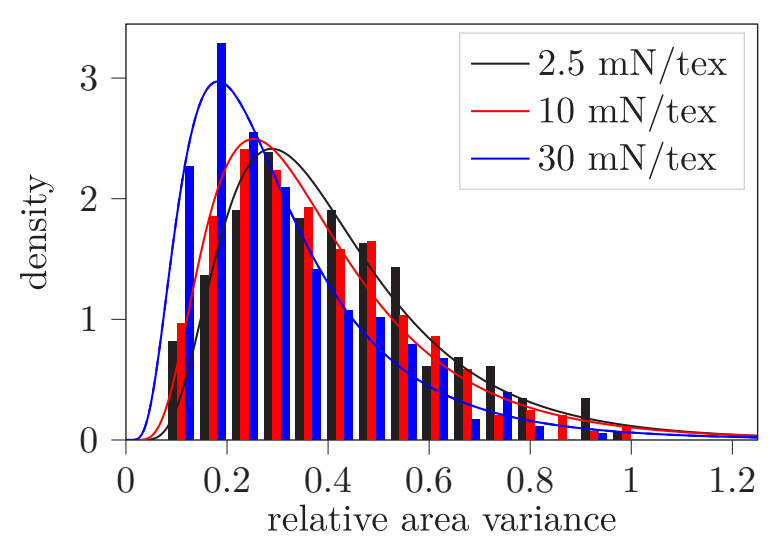

(c)

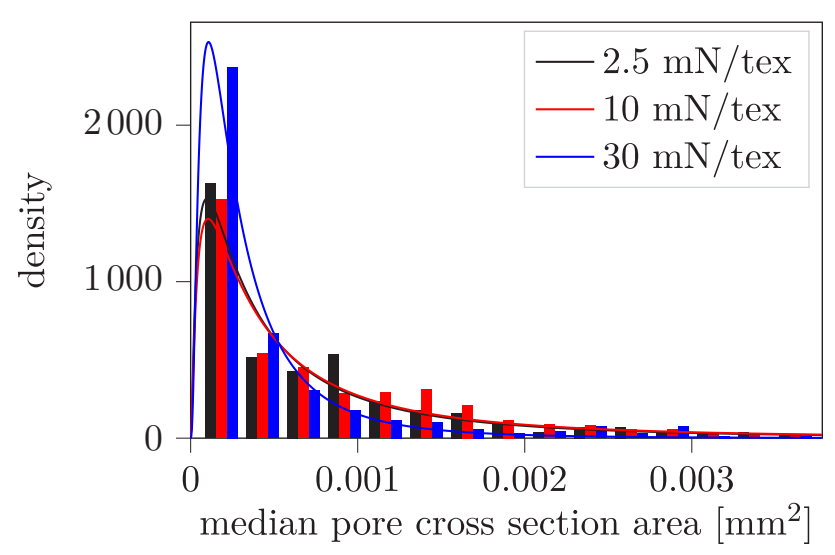

(e)

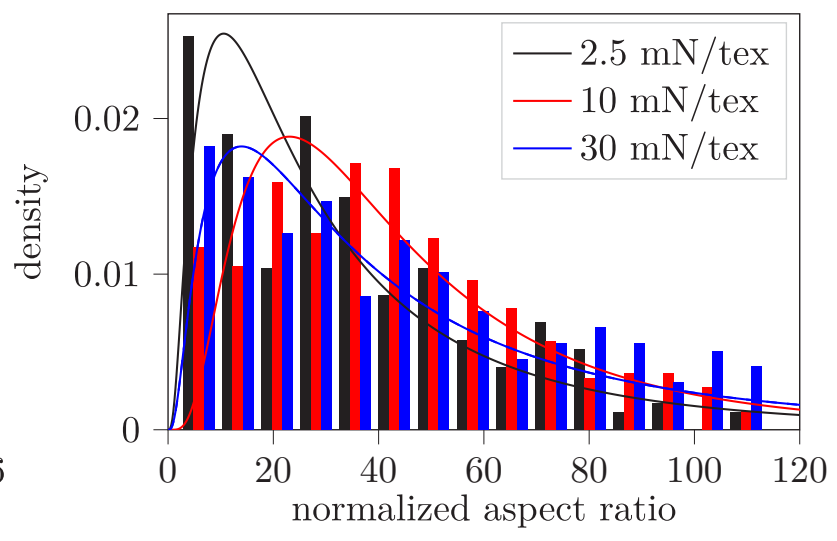

(b)

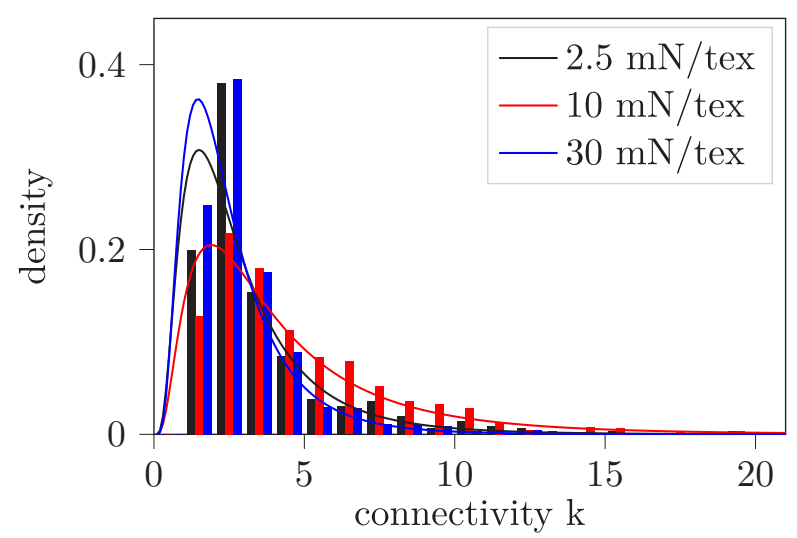

(d)

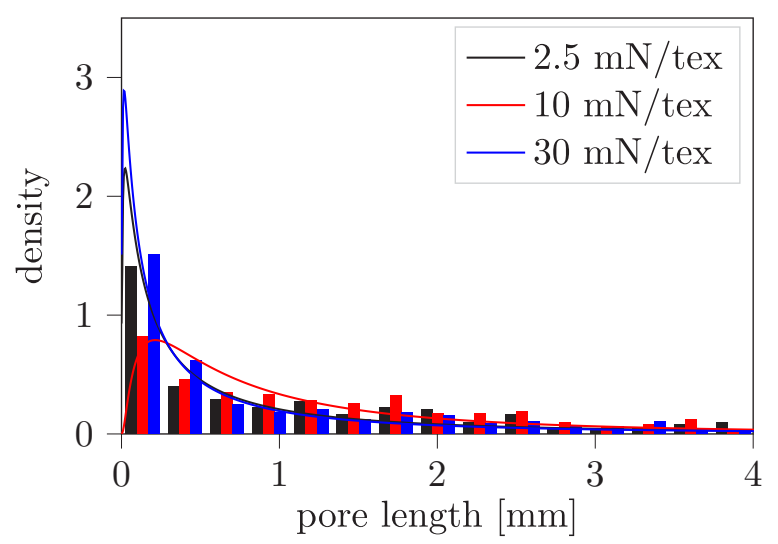

(f)

FIG. 5. Pore property distributions obtained from XTM void images segmented with a restricted watershed: (a) median shape factor, (b) aspect ratio, (c) relative area variance, (d) connectivity, (e) median cross-section area, and (f) pore arc length. Distributions have been fitted to a log-normal distribution.

waiting times in pores with high aspect ratio, such as yarn pores, directly translate to the observed uptake behavior at a macroscale.

\section{Analysis of volume flux and free (surface) energy rates}

Figure 8 gives a color-coded 3D visualization of the uptake in sample $3 / \mathrm{III}$, with $2.5 \mathrm{mN} /$ tex, for which the volume flux is shown by the black curve in Fig. 7(b). During the initial waiting period in Figs. 8(a) and 8(b) $(t<200$ s) only a little water is taken up, primarily as corner films. In Figs. 8(c)-8(j), capillary uptake happens as a series of filling events of long interfiber pore spaces. Already at $t=607 \mathrm{~s}$ [Fig. 8(e)], water is found all along the yarn and has reached the top of the FOV; filling is probably ongoing above the FOV, while large parts of the yarn inside the FOV still remain unfilled. The 


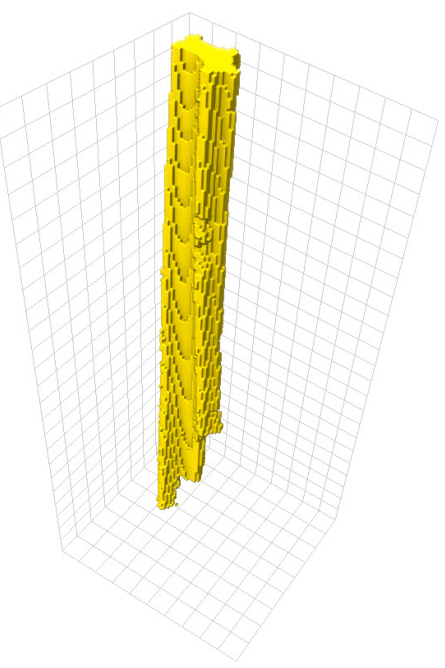

(a)

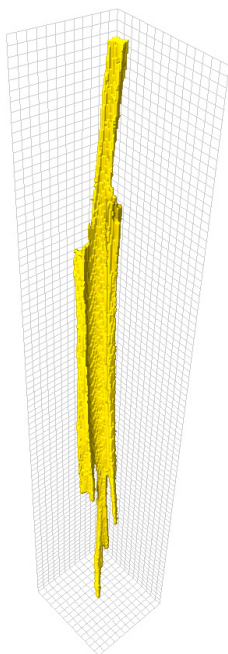

(b)

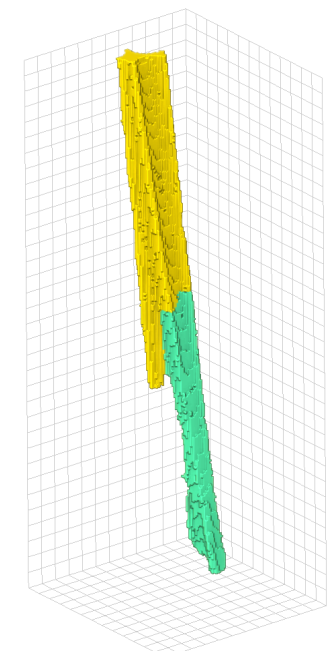

(c)

FIG. 6. (a) Example of a regular pore, (b) example of an irregular pore, and (c) example of a pore connection displayed on a $22-\mu \mathrm{m}$ (eight-pixel) grid.

filling events [peaks in Fig. 7(b)] correlate with the filling of long and narrow parts of the pore space, as indicated by the homogeneous color of these parts indicating the same filling time (Fig. 8). At the bottom of Figs. 8(i) and 8(j), an additional water dome (yellow bulky object) is visible that wets the yarn surface and slowly climbs on the yarn outer side. Figure 9(a) shows the time evolution of the water-air interface area $A_{w a}$, the water-solid area $A_{w s}$, the volume flux $Q$, and the resulting value for the free energy calculated according to Eq. (6) for the same sample, i.e., sample 3/III, with $2.5 \mathrm{mN} /$ tex. At early times $(t<200 \mathrm{~s}), A_{w a}$ and $A_{w s}$ grow slowly at an equal rate which is attributed to the corner films. The thickness of these corner films is at the limit of the spatial resolution and is only one or several pixels wide, not allowing us to accurately capture the triangular shape of the corner tips, but as flat film or just as a chain of disconnected small voxel clusters. While from geometric considerations of the corner tips one would expect $A_{w s}>A_{w a}$, we can only measure $A_{w s} \approx A_{w a}$. During the course of the uptake, $A_{w a}$ and $A_{w s}$ grow mostly continuously and finally reach a size of comparable magnitude. The large final value of $A_{w a}$ is attributed partly to pores at the yarn surface and partly to unsaturated pores. Figure 9(b)

TABLE II. Summary of fit data for all samples. Missing data are for nonconverging fits.

\begin{tabular}{|c|c|c|c|c|c|c|c|c|c|c|}
\hline \multirow[b]{2}{*}{ ID } & \multirow{2}{*}{$\begin{array}{l}\text { Tension } \\
(\mathrm{mN} / \text { tex })\end{array}$} & \multicolumn{3}{|c|}{ Earthquake $\Gamma$} & \multicolumn{4}{|c|}{ Generalized $\Gamma$} & \multicolumn{2}{|l|}{ Pareto } \\
\hline & & $C\left(\mathrm{~s}^{1-\delta}\right)$ & $\delta$ & $B(\mathrm{~s})$ & $\overline{\Delta t_{m}(\mathrm{~s})}$ & $d$ & $p$ & $\mu(\mathrm{s})$ & $x_{m}\left(10^{-15} \mathrm{~m}^{3} / \mathrm{s}\right)$ & $\bar{b}$ \\
\hline 3/III & 2.5 & 2.70 & 0.57 & 298.1 & 59.64 & 0.60 & 0.61 & 7.0 & 3.36 & 0.66 \\
\hline 4/ & 2.5 & & & & & & & & 3.88 & 0.49 \\
\hline $7 / \mathrm{II}$ & 2.5 & 3.11 & 0.43 & 183.6 & 25.28 & 0.55 & 0.54 & 7.0 & 2.77 & 0.42 \\
\hline 9/III & 2.5 & 4.11 & 0.51 & 457.6 & 90.77 & 0.72 & 0.77 & 8.3 & 3.09 & 0.69 \\
\hline \multicolumn{2}{|c|}{ average 2.5} & 3.31 & 0.50 & 313.1 & 58.56 & 0.62 & 0.64 & 7.43 & 3.28 & 0.57 \\
\hline $1 /$ & 10 & 1.07 & 0.89 & 311.5 & 103.35 & 0.70 & 0.49 & -9.9 & 3.25 & 0.9 \\
\hline $10 /$ & 10 & 1.09 & 1.03 & 47.8 & 51.55 & 1.06 & 1. & 7.0 & 3.88 & 0.49 \\
\hline $10 / \mathrm{III}$ & 10 & 2.08 & 0.68 & 716.2 & 116.84 & 0.48 & 0.47 & 11.9 & 3.88 & 0.53 \\
\hline 4/III & 10 & 2.18 & 0.63 & 445.4 & 71.42 & 0.49 & 0.45 & 7.0 & 3.37 & 0.48 \\
\hline $6 /$ & 10 & 1.23 & 0.96 & 155.7 & & & & & 3.08 & 0.58 \\
\hline $7 /$ & 10 & 4.93 & 0.16 & 187.3 & 13.33 & 0.60 & 0.63 & 6.8 & 3.25 & 0.49 \\
\hline $7 / \mathrm{III}$ & 10 & 1.60 & 0.80 & 247.8 & 115.05 & 0.67 & 0.65 & 8.95 & 3.33 & 0.77 \\
\hline \multicolumn{2}{|c|}{ average 10} & 2.03 & 0.74 & 301.7 & 78.59 & 0.67 & 0.63 & 5.29 & 3.44 & 0.61 \\
\hline $3 /$ & 30 & 1.77 & 0.70 & 52.8 & 24.45 & 0.74 & 0.74 & 5.0 & 3.25 & 0.53 \\
\hline 4/ & 30 & 2.49 & 0.49 & 130.0 & 20.25 & 0.50 & 0.48 & 6.9 & 3.37 & 0.48 \\
\hline $8 /$ III & 30 & 2.9 & 0.57 & 1661.1 & 11.71 & 0.45 & 0.26 & 14.0 & 3.53 & 0.68 \\
\hline $9 /$ III & 30 & 4.81 & 0.53 & 387.12 & 104.32 & 0.63 & 0.61 & 24.0 & 3.29 & 0.67 \\
\hline \multicolumn{2}{|c|}{ average 30} & 2.99 & 0.57 & 557.9 & 40.18 & 0.58 & 0.52 & 12.5 & 3.36 & 0.59 \\
\hline \multicolumn{2}{|c|}{ average all } & 2.58 & 0.64 & 377.3 & 62.15 & 0.63 & 0.60 & 8.0 & 3.37 & 0.59 \\
\hline
\end{tabular}




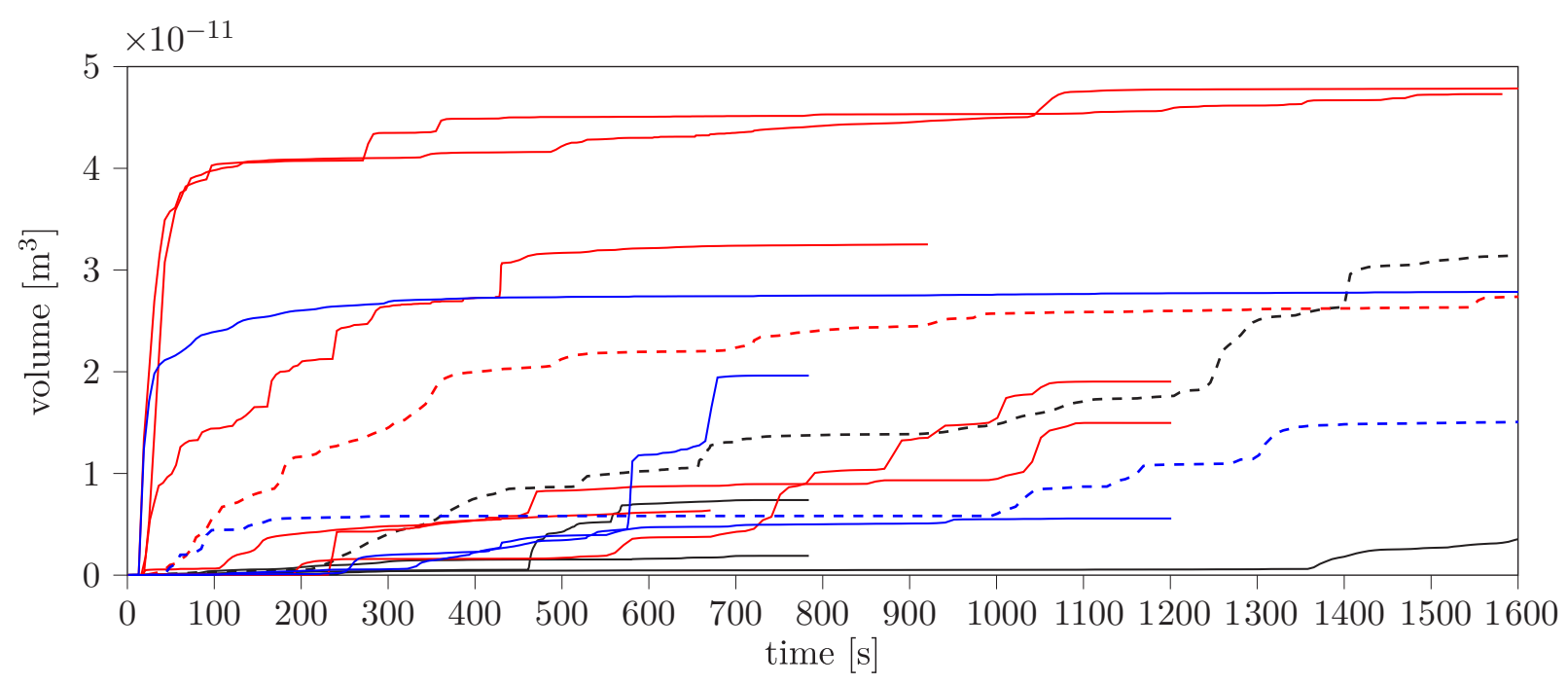

(a)

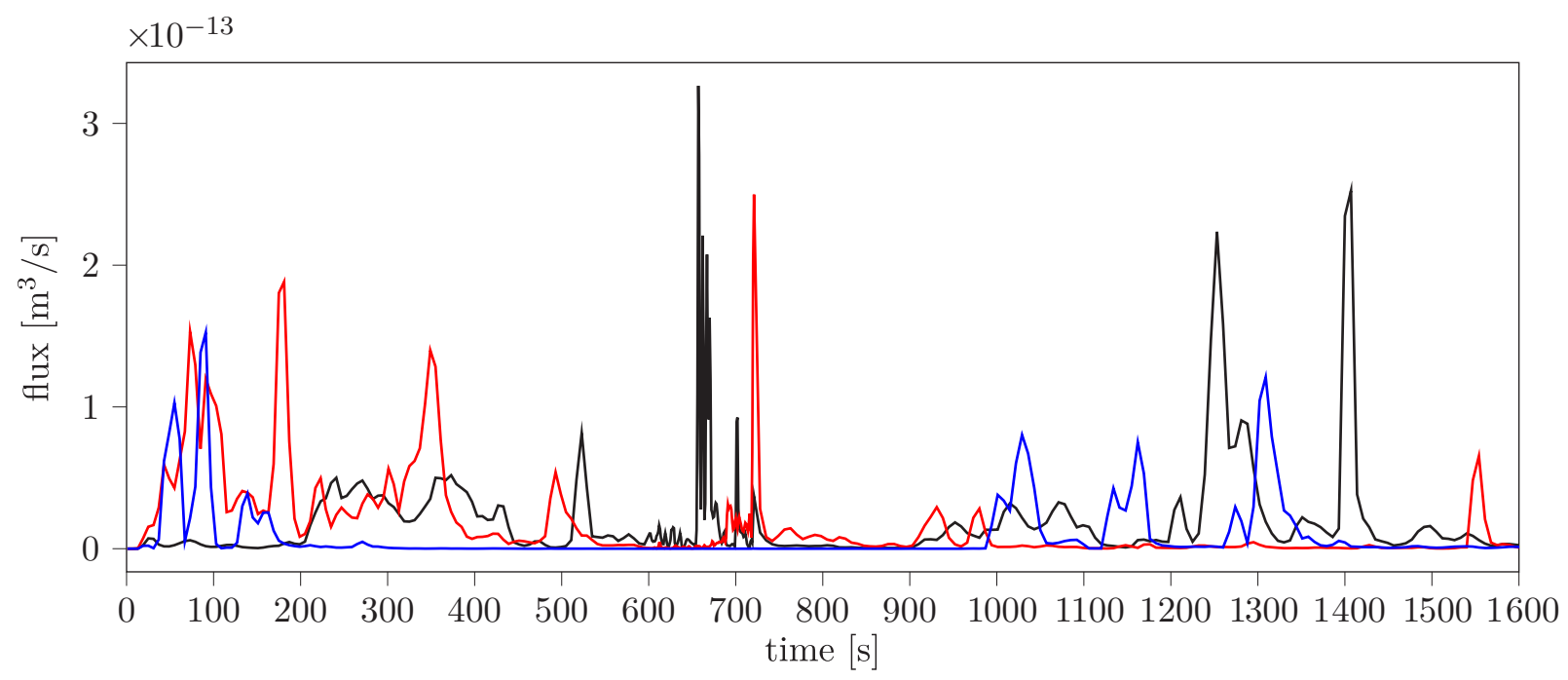

(b)

FIG. 7. (a) Volume uptake over time for all analyzed samples and (b) volume flux corresponding to three exemplary samples [highlighted as dashed lines in (a)]: black, 3/III, with $2.5 \mathrm{mN} /$ tex; red, 7/III, with $10 \mathrm{mN} /$ tex; and blue, 8/III, with $30 \mathrm{mN} /$ tex.

gives the rate of changes of interfaces over time. The growth of the wet surface $d A_{w s}$ always outweighs the growth of the water-air interface $d A_{w s}$, which leads to a continuous decrease of the free energy in Fig. 9(a). The peaks of volume flux coincide with drops in free energy shown in Fig. 9(a). The shape of the wet surface growth $d A_{w s}$ closely resembles the volume flux curve, while the water-air interface evolves rather independently, but mostly coincidentally with the wet surface and can even obtain negative values [Fig. 9(b)].

\section{Uptake process at pore scale}

To analyze the uptake process in single pores, the amount of water volume over time and the volume flux are determined at pore scale. It is found that the uptake process in a pore can happen in a single step or in several steps. The number of filling steps per pore are determined by counting the peaks in the pore flux. A peak is defined as any local flux maximum that exceeds 150 voxels $/ \mathrm{s} \approx 3.1 \times 10^{-6} \mathrm{~mm}^{3} / \mathrm{s}$, has a prominence, i.e., the vertical distance between the peak and its lowest contour line, of at least 75 voxels $/ \mathrm{s} \approx 1.6 \times$ $10^{-6} \mathrm{~mm}^{3} / \mathrm{s}$, and is at least $7 \mathrm{~s}$ away from the next higher peak (temporal resolution). The distribution of the number of filling events per pore for all pores in the 15 samples is given in Fig. 10. It is found that many pores are filled in a single-step pore-filling event, while others are filled in several steps, up to five steps and more.

\section{Example of pore filling occurring in a single event}

Figure 11 shows a typical single pore-filling event. The pore is a typical pore as presented in Fig. 6(a) (see Sec. IV A). The whole filling process takes around $5 \mathrm{~s}$ and is characterized by a single water-air interface steadily advancing from the 


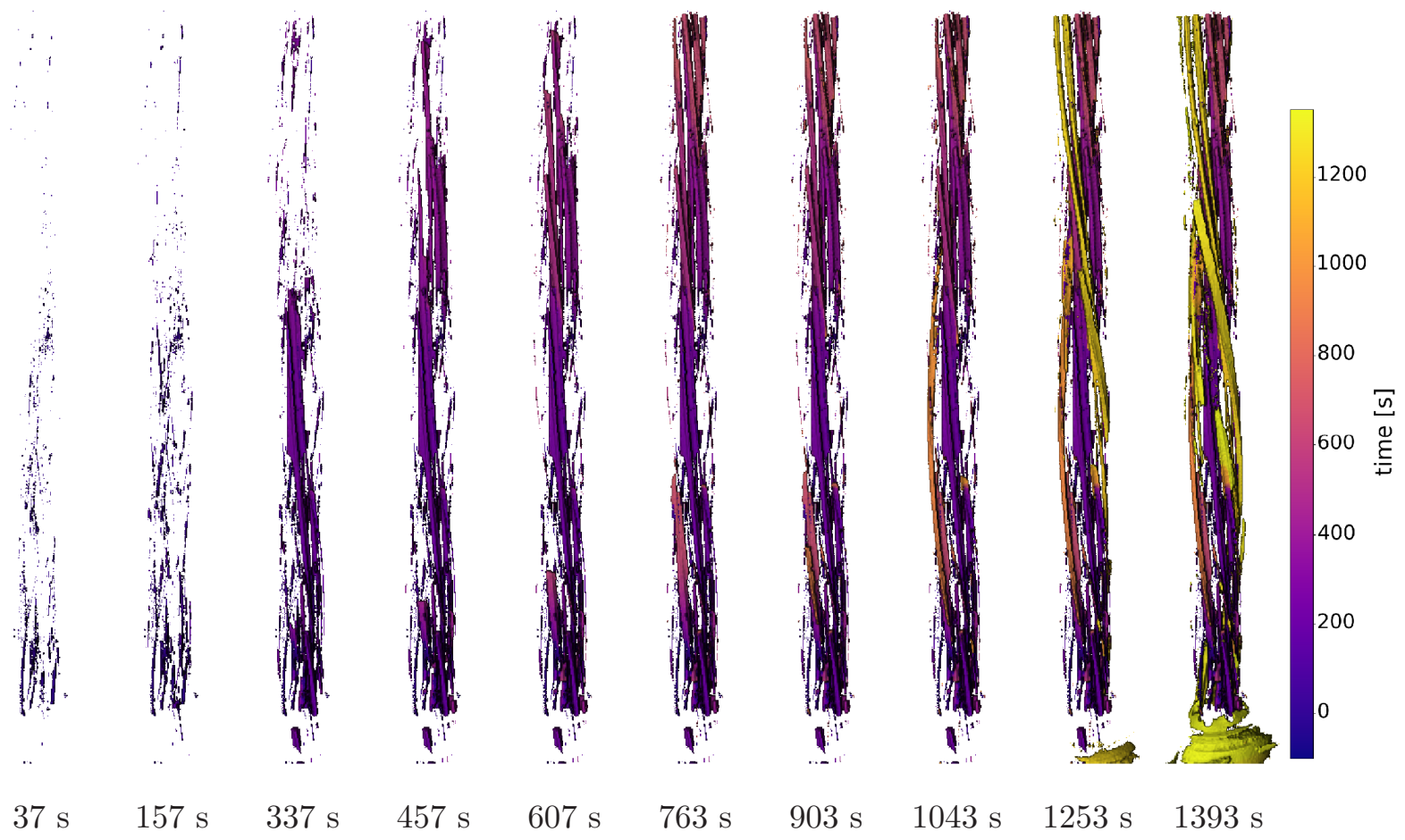

FIG. 8. The 3D false color visualization of water configuration inside the yarn at selected time steps (in seconds). The height of the displayed is $4 \mathrm{~mm}$.

pore inlet and moving steadily along. The interface includes fingers that wet the corners and whose tips are slightly ahead of the bulk water volume. The disconnectivity of water voxels in the corners comes from subresolution water films. After the filling event, the pore body is totally filled with water except for a small section at the bottom. As the pore has a rather regular cross-section area and shape over the height, the waterair interface does not grow much while the water-solid area increases when the interface rises in the pore. This process results in a sharp decrease of the free energy and a high volume flux. The average flow resistance $R$ during the filling event can be estimated knowing the free-energy gradient $d F / d t$ and volume flux $Q$. The total flow resistance is found in the range of $4 \times 10^{17} \mathrm{~Pa} \mathrm{~s} / \mathrm{m}^{3}$ calculated as an average over the whole filling event.

\section{Example of uptake in a pore in multiple steps}

Figures 12 and 13 document the typical multistep uptake process in a pore. We observed in Fig. 12(a) two small filling events at $t=370$ and $433 \mathrm{~s}$ and a large filling event at $t=658 \mathrm{~s}$. The two first events are characterized by a sharp increase in wet surface $A_{w s}$, while the water-air interface area $A_{w a}$ remains quite small, leading to an important decrease of free energy $F$. The total flow resistances $R$ for these events are calculated to $2.2 \pm 1.3 \times 10^{17}$ and $4.2 \pm 3.2 \times 10^{17} \mathrm{~Pa} \mathrm{~s} / \mathrm{m}^{3}$. We attribute the high uncertainty to the limit of the spatial and temporal resolution. Since the flow resistance $R$ is high, comparable to a 1-mm-long tube with a radius of $1.7 \mu \mathrm{m}$, and the decrease of $F$ not very steep, the volume fluxes remain rather low. Also, the analysis illustrates that the flow resistance stems from the upstream network and not from the much wider and shorter pore itself. The third filling event is characterized by important increases of both the waterair interface and wet surface, leading to a large and more importantly fast decrease in free energy. Although the flow resistance $R$ has a magnitude similar of those of the first two events at $0.78 \pm 10 \times 10^{17} \mathrm{~Pa} \mathrm{~s} / \mathrm{m}^{3}$, a much higher volume flux is observed due to the important decrease in free energy. Figures 12(b)-12(e) show the filling process in the pore, which has a particular shape. The pore shape shows four separate capillaries at the bottom that subsequently merge into a wide quadruple-conjoint capillary and then diverges again at the top into three capillaries. The pore filling starts from the bottom of the center left leg. The first filling step corresponds to the filling of a vertical pore segment [Fig. 12(c)] to a certain height and the second filling step corresponds to a further filling of this segment [Fig. 12(d)]. It appears from visual inspection that such filling events stop at the narrowest fiber gaps. After some waiting time, the interface gets remobilized and a large pore segment gets filled in a rapid burst during the third filling event [Fig. 12(e)]. The evolution of the water configuration before and during the third large filling event at $t \approx 658 \mathrm{~s}$ is analyzed in more detail in Fig. 13. During a long (approximately $200 \mathrm{~s}$ ) period before the filling event, the free energy slowly decreases while the water front also slowly advances [Figs. 13(b)-13(e)] until the water obtains a shape and configuration in the pore space that leads to a rapid filling at $t \approx 656 \mathrm{~s}$ [Fig. 13(f)]. The water configuration before the filling event is characterized as a partially filled pore that is limited by a narrowing of the pore space. Advancing past this narrowing into the pore opening comes at the cost of creating a new water-air interface and the effective free-energy release is small, allowing only a slow movement. The slow but 


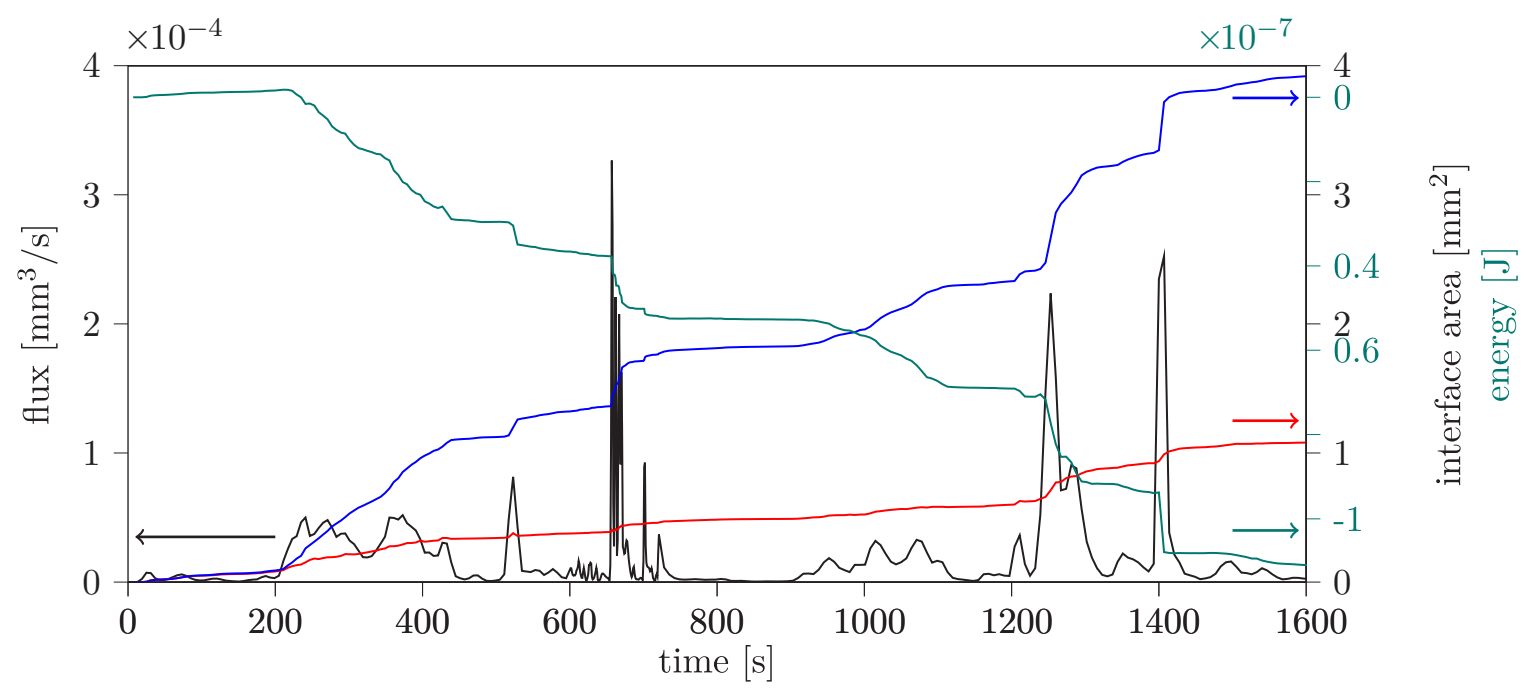

(a)

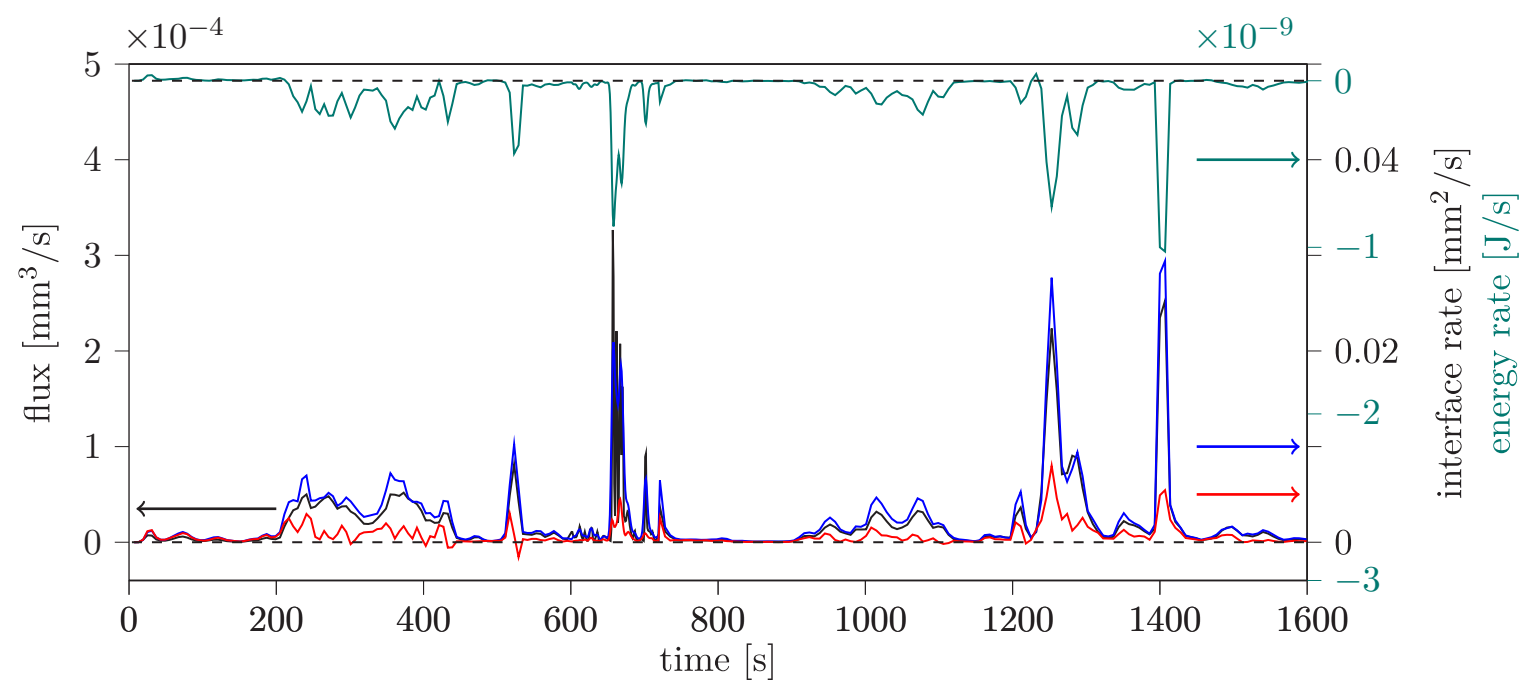

(b)

FIG. 9. (a) Volume flux $Q$ (black line) compared to the evolution of the wet surface $A_{w s}$ (blue line) and water-air interface $A_{w a}$ (red line) with the free energy $F$ (green line). (b) Volume flux $Q$ with interface and free-energy evolution rates. Colored arrows point to the respective axes.

persistent movement reconfigures the water until the interface reaches across the opening. This reconfiguration results in the slow decrease of free energy in Fig. 13. This reconfiguration process does not necessarily need the presence of a molecular precursor film or diffuse interface to explain the transition. The filling event itself shows the newly wet solid surface clearly outweighing the creation of a new water-air interface and therefore a strong release of free energy is observed.

\section{Interpore-filling process}

Additionally to the previously described intrapore-filling behavior, the interpore transition processes are of particular interest. Figure 14 illustrates the evolution of the water con- figuration at the connection of a pair of neighboring pores. There are early formed corner films present in both pores [dark blue in Fig. 14(b)], but we focus on the later pore to pore transition. The filling starts in the upper pore at around $670 \mathrm{~s}$ and some part is filled in the lower pore in the process. The water stops for roughly $25 \mathrm{~s}$ [Figs. 14(c) and 14(d)] before filling the remaining space of the lower pore at $t \approx 700 \mathrm{~s}$ [Fig. 14(e)]. We define this period as an interpore waiting time. The periods of low volume flux again correspond to a small decrease in free energy while high flux occurs simultaneously with a strong free-energy drop. The three detailed descriptions of pore-filling processes in different situations show the common behavior of fast filling events following waiting periods at nonobvious geometric restrictions. The analysis of the free energy reveals the physical origin of the 


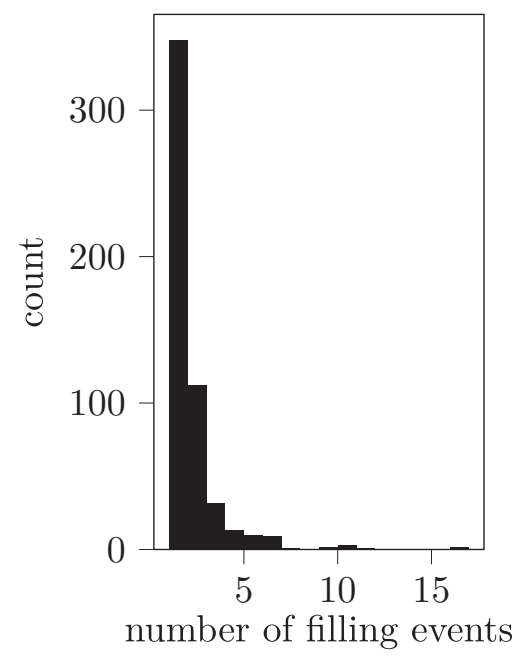

FIG. 10. Distribution of the number of filling events per pore for all examined samples and pores combined.

waiting times. Low flux is caused by a mutual annihilation of the contributions of the wet surface and water-air interface to the evolution of free energy. On the other hand, if more surface is wet than water-air interface created in pore sections with little cross-section variance, i.e., tubelike capillaries, the freeenergy gradient is large and high flux is observed as filling events.

\section{E. Pore-filling event sizes and waiting times}

Water transport in the investigated yarns displays successions of rapid filling events alternating with calmer periods. A phenomenon displaying such an abundance of repeated events warrants a careful study of its statistics. We provide statistical information about the size of pore-filling events and waiting times between these events using data from all yarn samples grouped per sample. The size of a pore-filling event is defined as the peak value of the flux during the filling of a pore as defined in Sec. IV D. The waiting time is defined as the time between flux peaks in the volume uptake curve of two neighboring pores counting both inter- and intrapore waiting times. The complementary cumulative distributions for porefilling event size and waiting time for the different tensions are presented in Fig. 15. Similar curves are observed for the three tension values. The filling size distribution is skew with a long end tail towards large sizes. The average flux event size is around $3.3 \times 10^{-14} \mathrm{~m}^{3} / \mathrm{s}$, the standard deviation is $7.0 \times 10^{-14} \mathrm{~m}^{3} / \mathrm{s}$, and extreme values go up to $77.7 \times 10^{-14}$ $\mathrm{m}^{3} / \mathrm{s}$ while they cover a range of $10^{2}$.

This means that extreme filling events may show a 20 times higher flux compared to the average flux. The waiting time distribution is also a skew distribution with an average of $122 \mathrm{~s}$, standard deviation of $193 \mathrm{~s}$, and extreme values up to $1475 \mathrm{~s}$ (25 min). This means that extreme waiting times may be up to 12 times longer than the average waiting time and they cover a range of $10^{3}$.

Overall, the distributions obtained show a noteworthy roughness and uncertainty and we attribute this to a combination of the limited size of the data set and the small number of pores per yarn in the FOV (fewer than 100). Also, we note that the end tails of the peak size distributions show some uncertainty, predominantly for $Q>5 \times 10^{-14} \mathrm{~m}^{3} / \mathrm{s}$. One reason is the limited number of extreme events, while the other is the uncertainty on high volume fluxes due to the limited time resolution. The temporal resolution is enhanced to $1 \mathrm{~s}$ only for a short time interval of 2 min during the total $30 \mathrm{~min}$ of acquisition, while for the remaining time the resolution is 6-7 s. To register a high flux or volume change per second, the pore has to have at least a volume of $5 \times 10^{-5} \mathrm{~mm}^{3}$ for the $1-\mathrm{s}$ time resolution and $35 \times 10^{-5} \mathrm{~mm}^{3}$ for the resolution of $7 \mathrm{~s}$, since the detectable flux cuts off at the pore volume divided by the time resolution. At the same instant, the free energy should show a sufficient gradient, which is less likely for larger pores since large pores show generally a smaller surface to volume ratio.

Such analysis allows a first estimation of the pressure evolution during spontaneous imbibition under the consideration of large experimental uncertainty. Employing the obtained resistance $R=4 \times 10^{17} \mathrm{~Pa} \mathrm{~s} \mathrm{~m}^{-3}$ and mean peak flux $Q=$ $3.3 \times 10^{-14} \mathrm{~m}^{3} / \mathrm{s}$, we get a capillary pressure of $p_{c}=R Q=$ $13.2 \mathrm{kPa}$ that corresponds to a tube with an equivalent radius of $7.3 \mu \mathrm{m}$ during the filling events. This radius is in the same range of a pore radius determined from measured cross sections of pores assuming a good representation of yarn pores as tubes. Estimating the pressure during the waiting times is more difficult because it is difficult to obtain a representative transported volume for these periods. Conservatively assuming that less than $30 \%$ of the pore volume $V$ is filled during the waiting times, we get an upper limit for the pressure of $p=0.3 R V(\Delta t)^{-1}=32 \mathrm{~Pa}$ for the median $V=3.22 \times 10^{-5} \mathrm{~mm}^{3}$ and $\Delta t=122 \mathrm{~s}$, which is by an order of magnitude smaller than during the filling events. While the fast filling events fall in line with the pore size's expected uptake rate, the overall uptake rate is overridden by the long interpore waiting times. To further analyze the distributions and the apparent dominance of rare extreme events, a Pareto distribution [see Eq. (7a)], a distribution similar to the generalized $\Gamma$ distribution as proposed by Corral [58] [see Eq. (7b)], and the standard definition of the generalized $\Gamma$ distribution [see Eq. (7c)] are fitted to the event size and waiting time data, respectively. The fitted parameters are provided in Table II. Here $x_{m}$ is the minimum possible value that scales the Pareto distribution. Given the very definition of a pore-filling event size peak (Sec. IV D), $x_{m}$ cannot obtain a value smaller than $3.1 \times 10^{-15} \mathrm{~m}^{3} / \mathrm{s}$ because this value is the minimum peak height defined in Sec. IVD. We do not observe a clear trend of the fitted parameters between the different samples or tension groups and $x_{m} \approx 3.4 \times 10^{-15} \mathrm{~m}^{3} / \mathrm{s}$ appears to be a characteristic value for this uptake process. The Pareto $b$ [Eq. (7a)] determines the shape of the distribution, a smaller value of $b$ means a flatter curve, and very small $b<1$ indicates heavier tails where more extreme values are indicated, i.e., it is $10^{1+b}$ times less likely to find a 10 times larger peak value. This is represented by the gradient $-b$ in the $\log _{10}$ plot in Figs. 15(d)-15(f). The $b$ varies from around 0.4 and up to 0.9 in one case, but overall a characteristic value of 0.6 is seen without a clear difference between the tension values. The parameters of the generalized $\Gamma$ distribution (7) fitted to the waiting times can be interpreted as follows. The $\mu$ is the offset, i.e., the minimum possible value or the time 


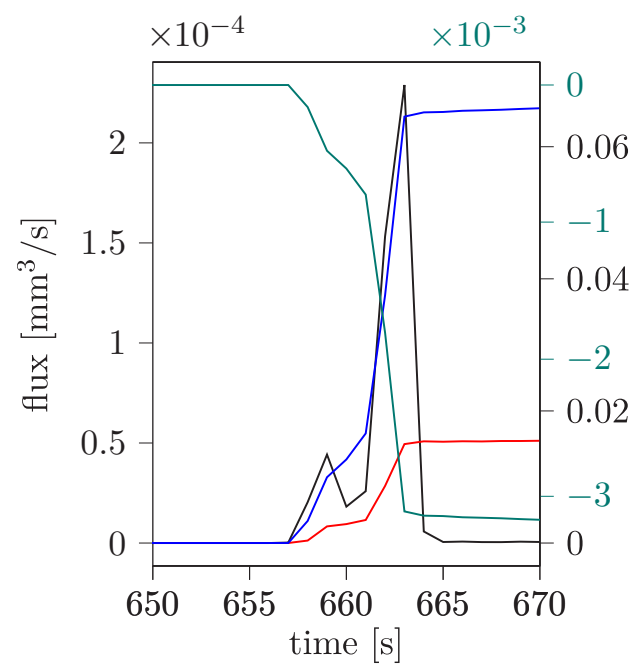

(a)

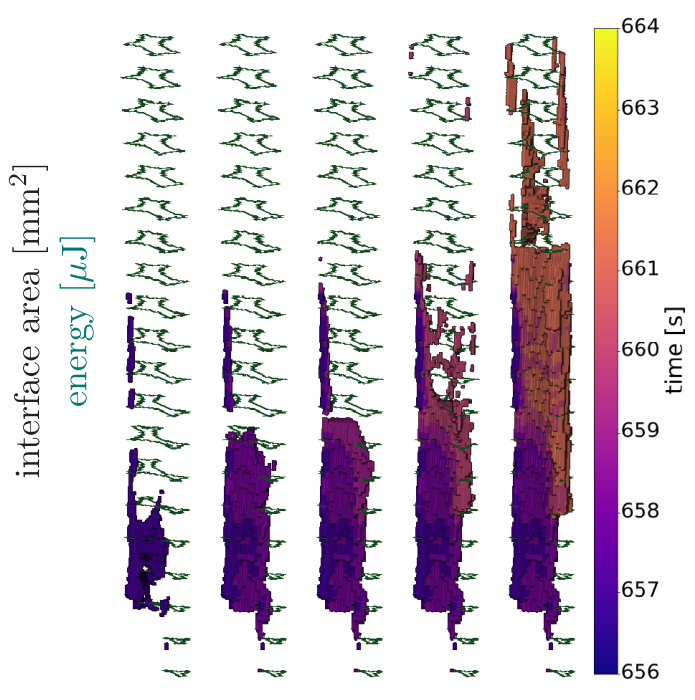

658 s 659 s 660 s 661 s 662 s
(b)
(c)
(d)
(e)
(f)

FIG. 11. (a) Water volume flux (black) and evolution rates for the wet surface (blue) and water-air interface (red) for the pore with a single step. (b)-(f) Visualization of the filling process with water in false color. Pore cross sections spaced by $27.5 \mu \mathrm{m}$ are in dark green.

resolution expected to be around $7 \mathrm{~s}$. The $\Delta t_{m}$ is the characteristic waiting time, which scales the distribution and equals 62 s. The interpretation of the shape parameters $d$ and $p$ is not trivial, but some useful conclusions can be drawn: If $d=p$, the Weibull distribution is recovered, which is commonly applied to lifetime analysis used in many disciplines.
Throughout our data set, $d \approx p$ suggests the waiting times to be the lifetime of quasistable water and interface configurations. Due to $d<1$ and $p<1$, the variance is inherently high.

We then use a second analysis of the distribution which was proposed by Corral [58] to fit the recurrence time of

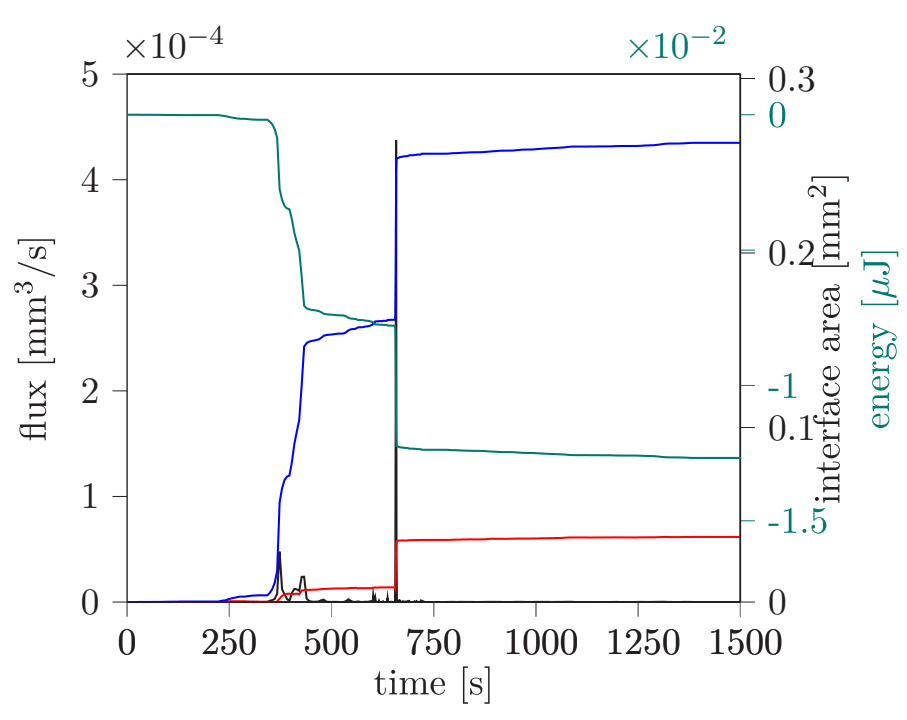

(a)

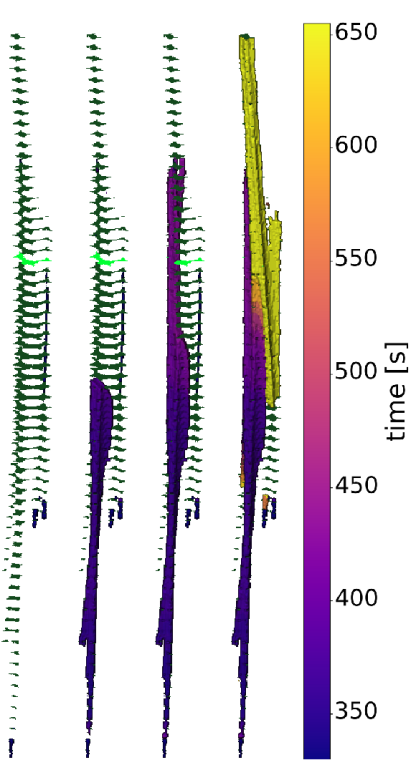

$330391433661 \mathrm{~s}$

(b) (c) (d) (e)

FIG. 12. (a) Comparison of the interface area evolution (blue, wet surface; red, water-air interface) to the flux (black) for a pore with three filling events. (b)-(e) Water configuration in false color before and after the flux peaks at selected time steps in seconds. The green shows the outline of the pore spaced by $27.5 \mu \mathrm{m}$. 


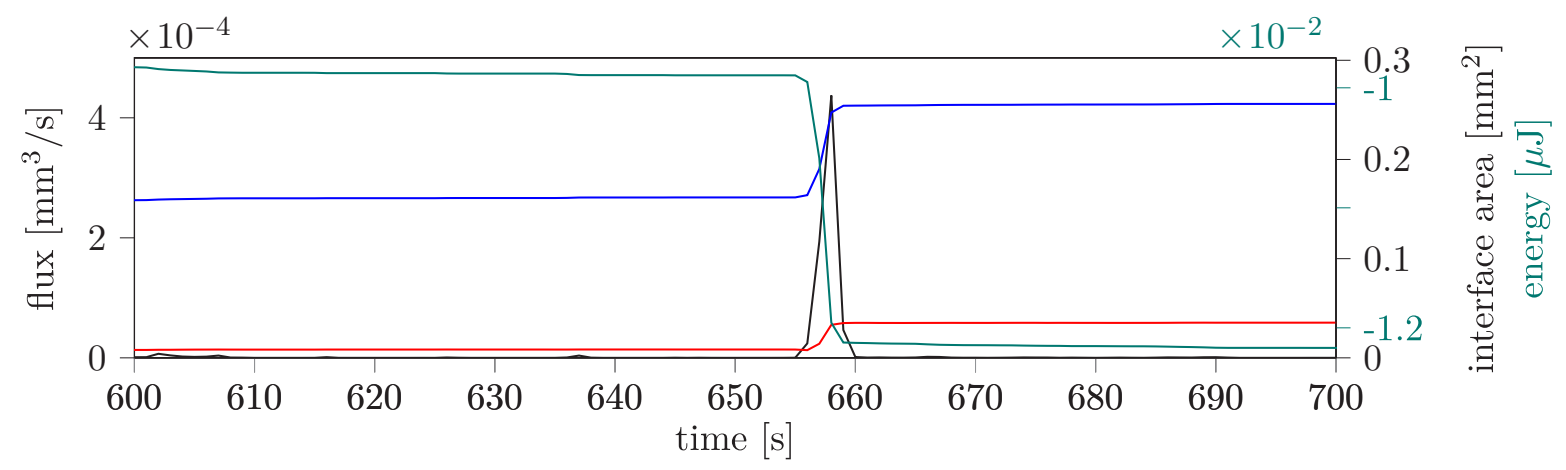

(a)
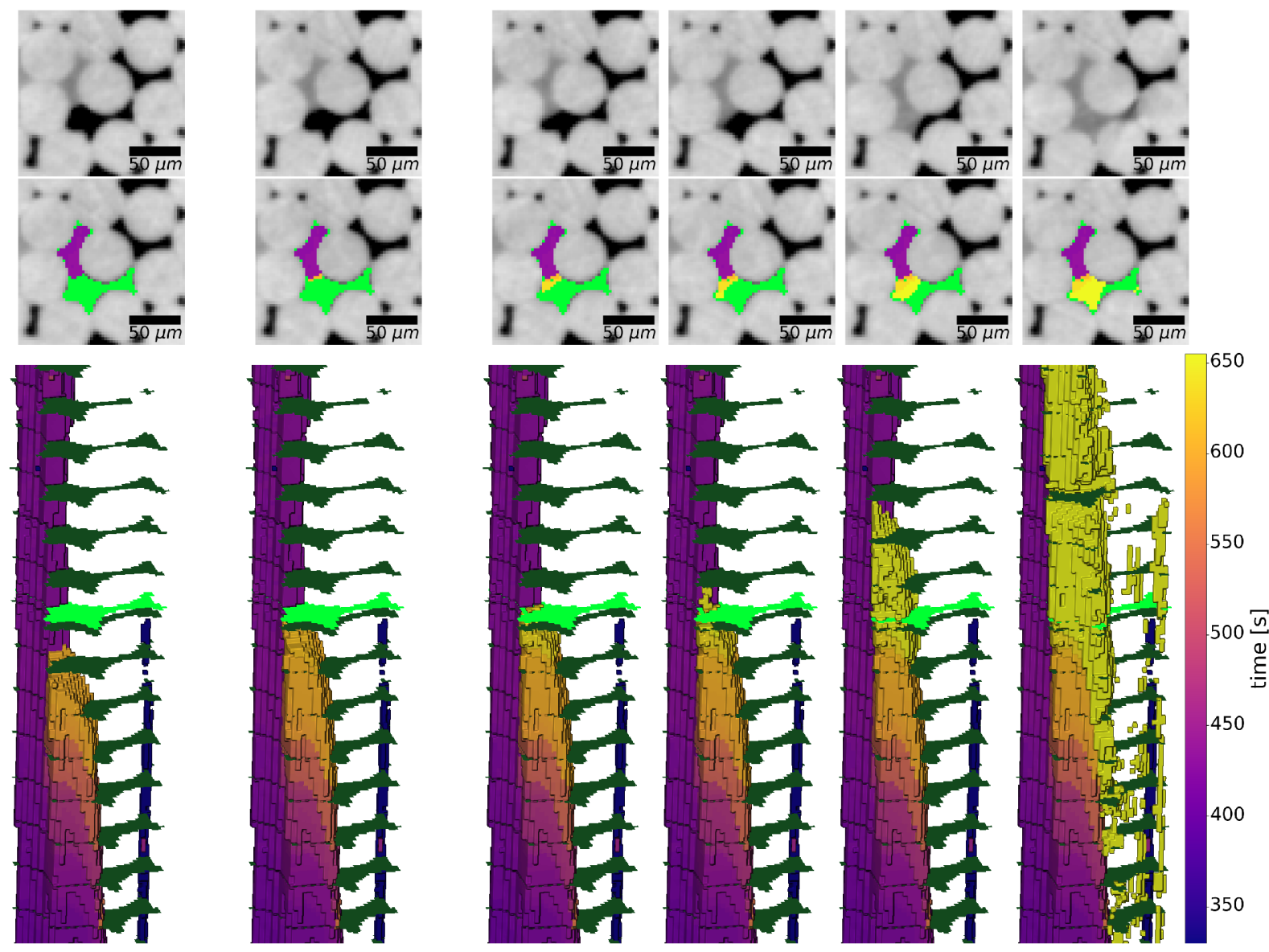

$601 \mathrm{~s}$

$621 \mathrm{~s}$

$641 \mathrm{~s}$

$655 \mathrm{~s}$

$656 \mathrm{~s}$

$657 \mathrm{~s}$

(b)

FIG. 13. (a) Evolution of interface areas (blue, wet surface; red, water-air interface) with corresponding free energy (green). (b) Visualization of the corresponding water configuration at selected time steps. The dark green slices in the 3D projection outline the pore geometry and are spaced by $27.5 \mu \mathrm{m}$ (ten pixels). The bright green slice highlights the position of the displayed cross section. The raw image is shown on top in grayscale. The color coding denotes the time when water reaches a location: purple labeled water entered the pore before $t=500 \mathrm{~s}$ and orange to yellow capture the filling event. Dark green outlines the pore geometry.

earthquakes and apply Eq. (7b), which is an almost equivalent approximation of the generalized $\Gamma$ distribution in this parameter range [solid and dashed red lines in Figs. 15(a)-15(c)]. For small values of $\Delta t$, the distribution is basically a power law described by the exponent $1-\delta$, while the exponential factor comes in at a characteristic waiting time $B$ leading to a few but extremely long waiting times. The higher $B$ is, the later this decline occurs, enabling increased probability for medium waiting times. The factors $C$ and $\delta$ scale the distribution. Generally, for smaller $\delta$, the gradient of the distribution 


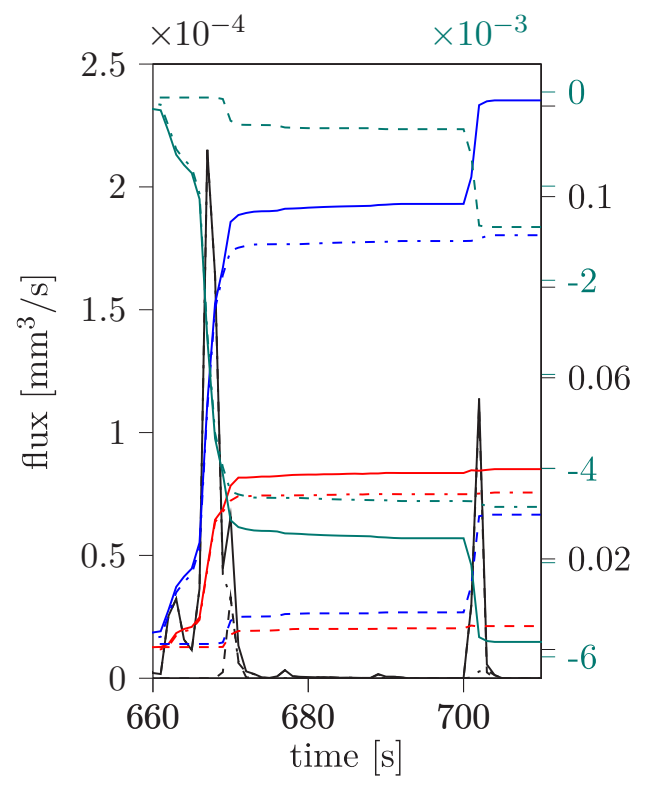

(a)

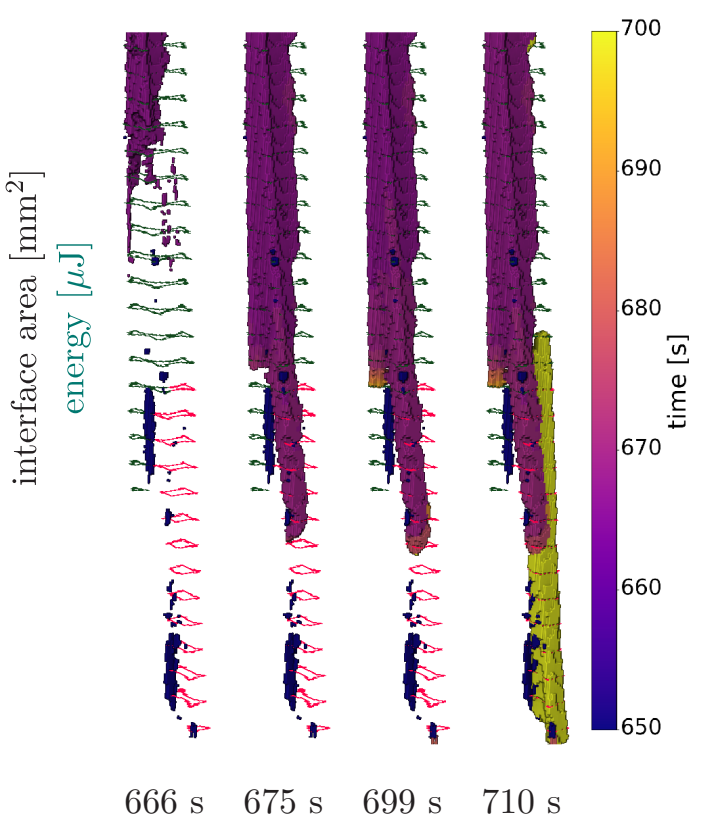

(b) (c) (d) (e)

FIG. 14. (a) Comparison of volume flux to interface and free-energy evolution of two neighboring pores combined (solid lines). Dashed (dash-dotted) lines correspond to the dark green (red) labeled pore. The top of the green pore is cropped to better visualize the contact area. Black shows the flux, red the water-air interface, blue the wet surface, and green the free energy. (b)-(e) Visualization of selected time steps with false colored water.

becomes steeper, making medium and long waiting times less likely:

$$
\begin{aligned}
F(x) & =\left(\frac{x}{x_{m}}\right)^{-b}, \\
F(\Delta t) & =\frac{C}{\Delta t^{1-\delta}} e^{-\Delta t / B}, \\
F(\Delta t) & =1-\frac{\gamma\left(\frac{d}{p},\left(\frac{\Delta t-\mu}{\Delta t_{m}}\right)^{p}\right)}{\Gamma\left(\frac{d}{p}\right)} .
\end{aligned}
$$

It is worthwhile to mention that the distributions of size and waiting time, describing the dynamics of spontaneous imbibition in yarns, show strong similarity to distributions describing other dynamical processes such as ruptures, landslides, and earthquakes, where the size distribution of events obeys a power (Pareto) law characterized by its exponent, called $b$ value in the earth sciences field. In general, these rupture processes are characterized by an accumulation of discrete events of size spreading over several orders of magnitude, also showing a characteristic distribution of recurrence times. As an example, the distribution of earthquake magnitudes $\left(\log _{10}\right.$ scale) is described by the Gutenberg-Richter law [59], which is closely related to the Pareto distribution used in our analysis at linear scale. The $b$ value is found to be commonly close to 1.0 with some variations around it (see, e.g., $[59,60])$. A value of 1 means that there will be 10 times more events with a magnitude $M$ or larger than events with a value equal to $M+1$ or larger. It is remarkable that we find $b$ values of around $0.4-0.9$ close to a $b$ value of 1 . In the field of earth science, stick-slip experiments conducted at lower scale in the laboratory or by simulation and earthquakes at large scale showing the same $b$ value are considered to refer to similar phenomena. Since these power laws show scale invariance and because of the similarities in the underlying physics, stick-slip experiments in the laboratory are considered as small-scale models of seismicity. The possible similarity between the size distribution of pore-filling events and rupture events is a point of interest for future research. Moreover, we also find a similarity in the distributions for waiting time with the distributions of interevents or recurrence times of earthquakes following also a power law, where commonly a generalized $\Gamma$ distribution is used [58], as in our analysis. We find an exponent $\delta$ around $0.5-0.7$, which approaches the $\delta$ value found for earthquakes, which is close to 0.67 [58]. Uptake in yarns does not show a uniform water front displacement in time, but is, just like rupture events, dominated by a few rare extreme fillings events [60-62].

\section{v. CONCLUSION}

We have used fast time-resolved synchrotron x-ray tomographic microscopy to investigate spontaneous imbibition in yarns by temporally and spatially resolving the evolution of the water configuration. In addition to yielding qualitative insights from 4D imaging, the acquired data provide quantitative information on the imbibition dynamics. To understand the stepwise wicking behavior in yarns it is necessary to recall the general definition of the capillary pressure as the freeenergy gradient and consider its two degrees of freedom, the change in area of the wet surface, and the water-air interface. An analysis of the free-energy rate can be obtained from the image data by extracting the interface areas. 


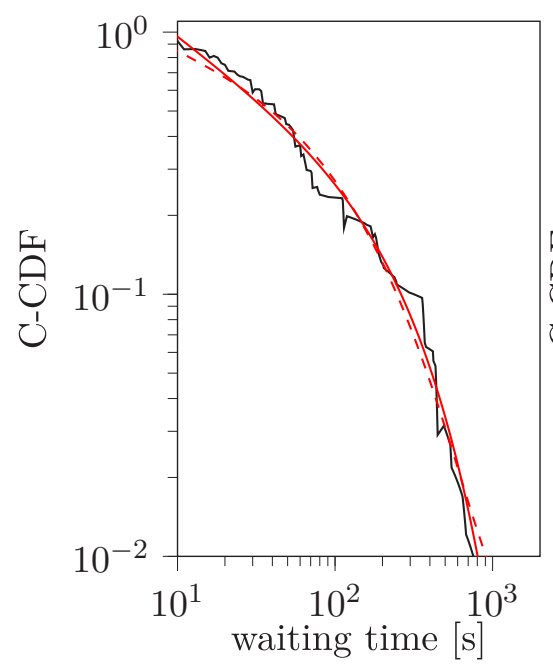

(a)

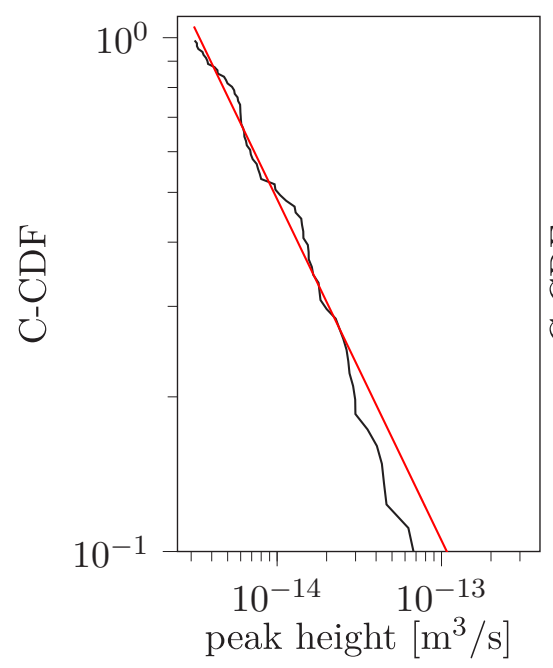

(d)

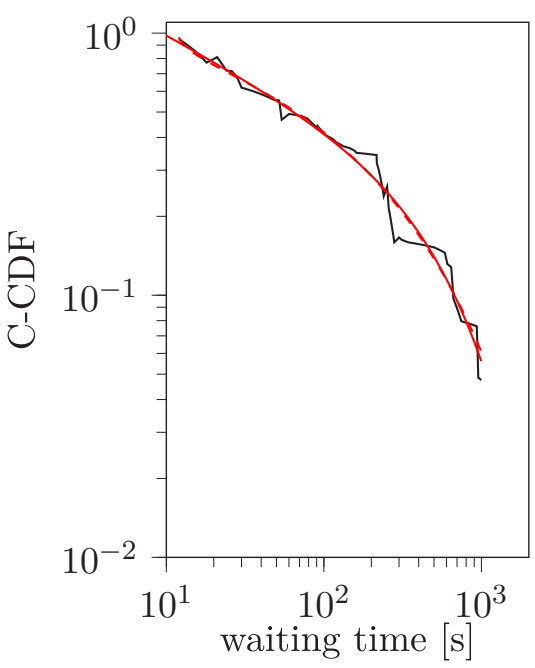

(b)

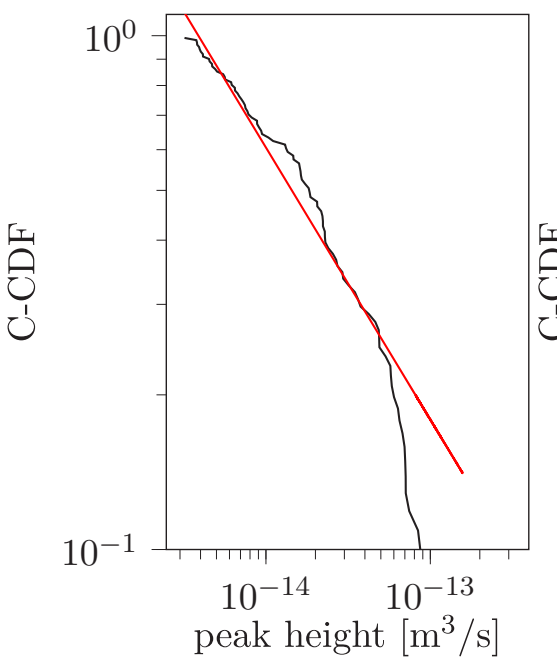

(e)

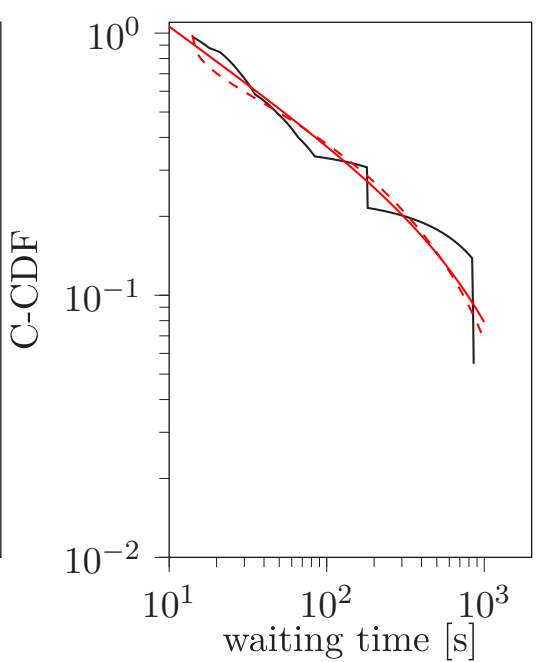

(c)

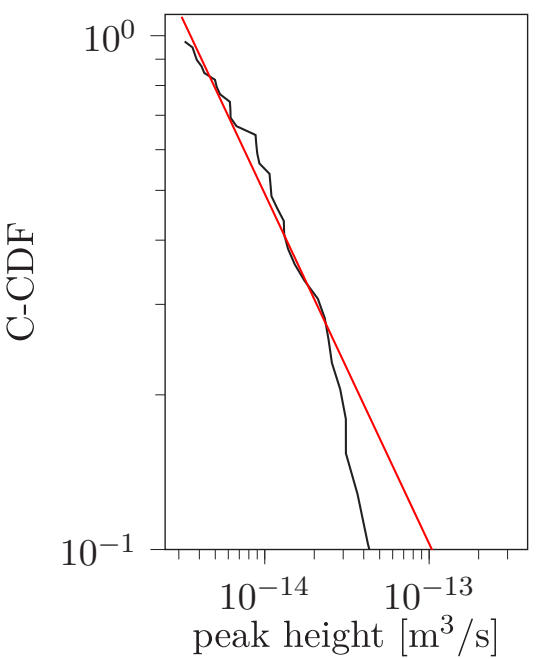

(f)

FIG. 15. (a)-(c) Waiting time distribution (black solid line) with fitted earthquake $\Gamma$ (red solid line) and generalized $\Gamma$ distribution fit (red dashed line) and (d)-(f) maximum volume flux distribution (black line) and fitted Pareto function (red line) for samples (a) and (d) $3 /$ III, with $2.5 \mathrm{mN} /$ tex, (b) and (e) 10/III, with $10 \mathrm{mN} /$ tex, and (c) and (f) $8 / \mathrm{III}$, with $30 \mathrm{mN} /$ tex.

Short periods in the range of seconds of high flux and high free-energy release are separated by waiting times up to several hundreds of seconds of low flux. Rapid filling events are seen to change little at the water-air interface but wetting large portions of the solid pore surface. The free-energy gradient (which is equal to the capillary pressure) resulting from this water configuration can readily overcome the resistance to flow posed by the yarn upstream. As such, the rapid filling events present familiar characteristics, such as what is seen in capillary tubes. On the other hand, the water can come to a halt where very little flow would happen over comparatively long times. Such standing-still situations were actually revealed to be the locus of intense microreorganization of the water configuration. At such places, the voids between the fibers would not provide a readily accessible path for wetting, resulting in the free-energy gradient to approach zero. The waiting times can be described as the lifetimes of quasistable water configurations with almost vanishing free-energy gradient. Eventually, the solid-water-air interfaces can reestablish a fast wetting configuration through the complex merging pores and flow can resume. The insightful imaging of this slow reorganization provides a delightful direct observation of the persistence of water wetting. The variability of both flux and waiting times spreads over several orders of magnitude. The consequence is a stepwise transport between quasiequilibria that results in the particular wicking dynamics. A topic for future research is the analysis of the striking similarity between the dynamics of such sudden pore-filling events in yarns and those of earthquakes and other stick-slip dynamics to make use of the characteristic distribution of high flux and waiting periods in a pore network model.

\section{ACKNOWLEDGMENTS}

We thank Monosuisse AG for providing the fibers. We would like to thank Sascha Krügl, LPMT at Université 
de Haute-Alsace, and Stefan Carl, Laboratory for Building Physics, EMPA, for the design and construction of the sample setup. We also acknowledge the provision of facilities and support during the sample production by Sascha Krügl, Mathieu Decrette, and Marie-Ange Bueno at LPMT. We further acknowledge the Paul Scherrer Institut, Villigen, Switzerland for provision of synchrotron radiation beamtime at the TOMCAT beamline X02DA of the SLS.

\section{APPENDIX}

\section{Plasma-enhanced chemical vapor deposition on PET filaments}

At first, the PET filaments are cleaned from spin finish residuals in a roll-to-roll dip coater using a washing solution with soap. After washing, $250 \mathrm{~m}$ of filament is wound around the drum electrode of a pilot-scale roll-to-roll plasma chamber. The chamber is evacuated to $3 \times 10^{-4} \mathrm{~Pa}$. The drum rotates at a speed $2 \mathrm{~m} / \mathrm{min}$, i.e., about one turn per minute. Plasma cleaning and etching occur prior to coating; a gas mixture of $\mathrm{Ar}$ and $\mathrm{O}_{2}$ is introduced at flow rates of 160 and 40 SCCM (SCCM denotes cubic centimeter per minute at STP). The plasma driving radio-frequency (rf) power is held at $400 \mathrm{~W}$ for $2 \mathrm{~min}$. The gas pressure for all steps is $10 \mathrm{~Pa}$ and the chamber is evacuated between steps. For the deposition step, the reactive gas mixture of $\mathrm{CO}_{2}$ and $\mathrm{C}_{2} \mathrm{H}_{4}$ is introduced from two gas spreading systems, each at flow rates of $2 \times 200$ and $2 \times 50$ SCCM, respectively. The applied rf power is set to $700 \mathrm{~W}$ for $20 \mathrm{~min}$ to deposit a 30 -nm-thick $\mathrm{C}: \mathrm{H}: \mathrm{O}$ plasma polymer film covering the entire filament surface. Subsequently, the $\mathrm{CO}_{2}$ gas flow rate is raised to $2 \times 300$ SCCM for $1 \mathrm{~min}$, maintaining other parameters to terminate the coating with a more-oxygen-rich outermost layer. This results in a highly permanent coating while enhancing the wettability. The deposition process is monitored for steady plasma conditions using a voltage-current probe. Reference flat PET plates coated under equal PECVD conditions show a resulting Young contact angle with water in air of $48^{\circ} \pm 5^{\circ}$ measured 1 day after deposition, that is, after potential restructuring processes of the plasma coating have been saturated. The filament is subsequently carefully handled to maintain the surface properties.

\section{Derivation of Washburn's law from Darcy's law (4)}

The free-energy gradient in a circular tube is a constant and can be written as

$$
\begin{aligned}
\frac{d F}{d V} & =\frac{\gamma_{w a}\left(d A_{w a}-d A_{w s} \cos \vartheta\right)}{d V}, \\
d A_{w a} & =0,
\end{aligned}
$$

$$
\begin{aligned}
d A_{w s} & =2 \pi r d h, \\
d V & =\pi r^{2} d h, \\
\frac{d F}{d V} & =-\frac{2 \gamma_{w a} \cos \vartheta}{r} .
\end{aligned}
$$

Note that this expression only appears equivalent to the Laplace pressure of a hemisphere, but the capillary force results from the wet perimeter and not the shape of the water-air interface. Laminar flow is assumed and the resistance $R$ can be obtained from Poiseuille's law with the imbibition height $h$ as the tube length:

$$
R_{p}=\frac{8 \eta h}{\pi r^{4}}
$$

Inserting (A1) and (A2) into (4) yields Washburn's square root law over time:

$$
\begin{aligned}
-\frac{d F}{d V} & =R \frac{d V}{d t}, \\
h \frac{d h}{d t} & =\frac{\gamma_{w a} r \cos \vartheta}{4 \eta}, \\
h & =\sqrt{\frac{\gamma_{w a} r \cos \vartheta}{2 \eta} t} .
\end{aligned}
$$

Equations (A2) and (A3) can be modified to include a constant upstream resistance $R_{e}$ :

$$
\frac{2 \gamma_{w a} \cos \vartheta}{r}=\left(R_{e}+\frac{8 \eta h}{\pi r^{4}}\right) \pi r^{2} \frac{d h}{d t} .
$$

While this differential equation can be solved,

$$
h(t)=-\frac{R_{e} \pi r^{4}}{8 \eta}+\sqrt{\left(\frac{R_{e} \pi r^{4}}{8 \eta}\right)^{2}+\frac{\gamma_{w a} r \cos \vartheta}{2 \eta} t},
$$

the limiting cases are most interesting

$$
h \approx \begin{cases}\frac{2 \gamma_{w a} \cos \vartheta}{R_{e} \pi r^{3}} t & \text { for } R_{e} \gg R_{p} \\ \sqrt{\frac{\gamma_{w a} r \cos \vartheta}{2 \eta} t} & \text { for } R_{e} \ll R_{p},\end{cases}
$$

showing a linear uptake versus time for high entry resistance and Washburn's equation for a pore resistance much higher than the entry resistance.
[1] E. Kissa, Text. Res. J. 66, 660 (1996).

[2] P.-G. de Gennes, F. Brochard-Wyart, and D. Quere, Capillarity and Wetting Phenomena: Drops, Bubbles, Pearls, Waves (Springer, New York, 2013).

[3] D. Quéré, Europhys. Lett. 39, 533 (1997).

[4] J. Schoelkopf, P. A. C. Gane, C. J. Ridgway, and G. P. Matthews, Colloids Surf. A 206, 445 (2002).
[5] M. Parada, X. Zhou, D. Derome, R. M. Rossi, and J. Carmeliet, Text. Res. J. 89, 3519 (2018).

[6] A. Ashari, T. M. Bucher, H. V. Tafreshi, M. A. Tahir, and M. S. A. Rahman, Int. J. Heat Mass Transf. 53, 1750 (2010).

[7] K. Vandersteen, J. Carmeliet, and J. Feyen, Transp. Porous Media 50, 197 (2003). 
[8] T. Agaesse, A. Lamibrac, F. N. Büchi, J. Pauchet, and M. Prat, J. Power Sources 331, 462 (2016).

[9] V. Joekar-Niasar, M. Prodanović, D. Wildenschild, and S. M. Hassanizadeh, Water Resour. Res. 46, W06526 (2010).

[10] V. Joekar-Niasar, S. M. Hassanizadeh, and H. K. Dahle, J. Fluid Mech. 655, 38 (2010).

[11] P. Van Marcke, B. Verleye, J. Carmeliet, D. Roose, and R. Swennen, Transp. Porous Media 85, 451 (2010).

[12] J. Zhao, F. Qin, D. Derome, and J. Carmeliet, J. Hydrol. 588, 125080 (2020).

[13] J. Zhao, F. Qin, D. Derome, Q. Kang, and J. Carmeliet, Adv. Water Resour. 145, 103738 (2020).

[14] T. Lerouge, O. Pitois, D. Grande, B. Le Droumaguet, and P. Coussot, Soft Matter 14, 8137 (2018).

[15] M. Y. I. Parada, C. M. Schlepütz, R. M. Rossi, and D. Derome, J. Carmeliet, Text. Res. J. 89, 4967 (2019).

[16] M. Parada, P. Vontobel, R. M. Rossi, D. Derome, and J. Carmeliet, Transp. Porous Media 119, 611 (2017).

[17] N. R. S. Hollies, M. M. Kaessinger, and H. Bogaty, Text. Res. J. 26, 829 (1956).

[18] N. R. S. Hollies, M. M. Kaessinger, B. S. Watson, and H. Bogaty, Text. Res. J. 27, 8 (1957).

[19] A. B. Nyoni and D. Brook, J. Text. Inst. 97, 119 (2006).

[20] A. Patnaik, R. S. Rengasamy, V. K. Kothari, and A. Ghosh, Text. Prog. 38, 1 (2006).

[21] M. M. Hossain, D. Hegemann, A. S. Herrmann, and P. Chabrecek, J. Appl. Polym. Sci. 102, 1452 (2006).

[22] Y. Li, R. Fischer, R. Zboray, P. Boillat, M. Camenzind, C. Toncelli, and R. M. Rossi, ACS Appl. Mater. Interfaces 12, 29908 (2020).

[23] Z. Sadjadi, M. Jung, R. Seemann, and H. Rieger, Langmuir 31, 2600 (2015).

[24] H. Mehrabian, P. Gao, and J. J. Feng, Phys. Fluids 23, 122108 (2011).

[25] F. Moebius and D. Or, J. Colloid Interface Sci. 377, 406 (2012).

[26] A. Ferrari and I. Lunati, Adv. Water Resour. 74, 1 (2014).

[27] J. Zhao, Q. Kang, J. Yao, H. Viswanathan, R. Pawar, L. Zhang, and H. Sun, Water Resour. Res. 54, 1295 (2018).

[28] F. Qin, L. Del Carro, A. Mazloomi Moqaddam, Q. Kang, T. Brunschwiler, D. Derome, and J. Carmeliet, J. Fluid Mech. 866, 33 (2019).

[29] H. S. Rabbani, V. Joekar-Niasar, and N. Shokri, J. Colloid Interface Sci. 473, 34 (2016).

[30] K. Singh, H. Scholl, M. Brinkmann, M. D. Michiel, M. Scheel, S. Herminghaus, and R. Seemann, Sci. Rep. 7, 444 (2017).

[31] Q. Lin, B. Bijeljic, S. Berg, R. Pini, M. J. Blunt, and S. Krevor, Phys. Rev. E 99, 063105 (2019).

[32] A. Piovesan, T. Van De Looverbosch, P. Verboven, C. Achille, C. Parra Cabrera, E. Boller, Y. Cheng, R. Ameloot, and B. Nicolai, Lab Chip 20, 2403 (2020).

[33] G. Zhang, R. Parwani, C. A. Stone, A. H. Barber, and L. Botto, Langmuir 33, 12072 (2017).

[34] A. Mascini, V. Cnudde, and T. Bultreys, J. Colloid Interface Sci. 572, 354 (2020).

[35] A. AlRatrout, A. Q. Raeini, B. Bijeljic, and M. J. Blunt, Adv. Water Resour. 109, 158 (2017).

[36] I. Arganda-Carreras, V. Kaynig, C. Rueden, K. W. Eliceiri, J. Schindelin, A. Cardona, and H. S. Seung, Bioinformatics 33, 2424 (2017).
[37] T. Yu, J. Zhou, and M. Doi, Soft Matter 14, 9263 (2018).

[38] H. Goldstein, C. P. Poole, and J. L. Safko, Classical Mechanics: Pearson New International Edition (Pearson Education UK, Harlow, 2013).

[39] D. Patro, S. Bhattacharyya, and V. Jayaram, J. Am. Ceram. Soc. 90, 3040 (2007).

[40] P. Rupper, M. Vandenbossche, L. Bernard, D. Hegemann, and M. Heuberger, Langmuir 33, 2340 (2017).

[41] D. Hegemann, E. Lorusso, M. I. Butron-Garcia, N. E. Blanchard, P. Rupper, P. Favia, M. Heuberger, and M. Vandenbossche, Langmuir 32, 651 (2016).

[42] M. Stampanoni, A. Groso, A. Isenegger, G. Mikuljan, Q. Chen, A. Bertrand, S. Henein, R. Betemps, U. Frommherz, P. Böhler, D. Meister, M. Lange, and R. Abela, SPIE Proc. 6318, 63180M (2006).

[43] M. Buhrer, M. Stampanoni, X. Rochet, F. Buchi, J. Eller, and F. Marone, J. Synchrotron Radiat. 26, 1161 (2019).

[44] R. Mokso, C. M. Schleputz, G. Theidel, H. Billich, E. Schmid, T. Celcer, G. Mikuljan, L. Sala, F. Marone, N. Schlumpf, and M. Stampanoni, J. Synchrotron Radiat. 24, 1250 (2017).

[45] D. Paganin, S. C. Mayo, T. E. Gureyev, P. R. Miller, and S. W. Wilkins, J. Microsc. 206, 33 (2002).

[46] F. Marone and M. Stampanoni, J. Synchrotron Radiat. 19, 1029 (2012).

[47] C. R. Harris, K. J. Millman, S. J. van der Walt, R. Gommers, P. Virtanen, D. Cournapeau, E. Wieser, J. Taylor, S. Berg, N. J. Smith et al. Nature (London) 585, 357 (2020).

[48] S. van der Walt, J. L. Schönberger, J. Nunez-Iglesias, F. Boulogne, J. D. Warner, N. Yager, E. Gouillart, T. Yu, and scikit-image contributors, PeerJ 2, e453 (2014).

[49] S. Hoyer and J. J. Hamman, J. Open Res. Softw. 5, 10 (2017).

[50] P. Virtanen, R. Gommers, T. E. Oliphant, M. Haberland, T. Reddy, D. Cournapeau, E. Burovski, P. Peterson, W. Weckesser, J. Bright et al., Nat. Methods 17, 261 (2020).

[51] P. Thévenaz, U. E. Ruttimann, and M. Unser, IEEE Trans. Image Process. 7, 27 (1998).

[52] https://github.com/glichtner/pystackreg.

[53] J. Schindelin, I. Arganda-Carreras, E. Frise, V. Kaynig, M. Longair, T. Pietzsch, S. Preibisch, C. Rueden, S. Saalfeld, B. Schmid, J. Y. Tinevez, D. J. White, V. Hartenstein, K. Eliceiri, P. Tomancak, and A. Cardona, Nat. Methods 9, 676 (2012).

[54] B. Münch and L. Holzer, J. Am. Ceram. Soc. 91, 4059 (2008).

[55] B. Münch, P. Gasser, L. Holzer, and R. Flatt, J. Am. Ceram. Soc. 89, 2586 (2006).

[56] T. Lewiner, H. Lopes, A. W. Vieira, and G. Tavares, J. Graphics Tools 8, 1 (2012).

[57] G. Taubin, in Proceedings of IEEE International Conference on Computer Vision (IEEE Computer Society, New York, 1995), pp. 852-857.

[58] A. Corral, Phys. Rev. Lett. 92, 108501 (2004).

[59] J. Rivière, Z. Lv, P. A. Johnson, and C. Marone, Earth Planet. Sci. Lett. 482, 407 (2018).

[60] D. Amitrano, Eur. Phys. J.: Spec. Top. 205, 199 (2012).

[61] M. K. Sachs, M. R. Yoder, D. L. Turcotte, J. B. Rundle, and B. D. Malamud, Eur. Phys. J.: Spec. Top. 205, 167 (2012).

[62] D. Sornette and G. Ouillon, Eur. Phys. J.: Spec. Top. 205, 1 (2012). 